\title{
An interferometer experiment to explore the aspect angle dependence of stimulated electromagnetic emission spectra
}

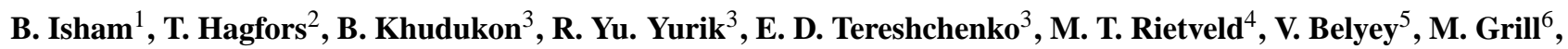 \\ C. $\mathrm{La} \mathrm{Hoz}^{5}$, A. Brekke ${ }^{5}$, and C. Heinselman ${ }^{7}$ \\ ${ }^{1}$ Interamerican University, Bayamón, Puerto Rico, USA \\ ${ }^{2}$ Max-Planck Institut für Sonnensystemforschung, Katlenburg-Lindau, Germany \\ ${ }^{3}$ Polar Geophysical Institute, Murmansk, Russia \\ ${ }^{4}$ EISCAT Scientific Association, Ramfjordbotn, Norway \\ ${ }^{5}$ University of Troms $\varnothing$, Troms $\varnothing$, Norway \\ ${ }^{6}$ Lancaster University, Lancaster, UK \\ ${ }^{7}$ SRI International, Menlo Park, California, USA
}

Received: 27 February 2004 - Revised: 1 December 2004 - Accepted: 5 January 2005 - Published: 31 January 2005

Part of Special Issue "Eleventh International EISCAT Workshop"

\begin{abstract}
When the Earth's ionosphere is irradiated by a radiofrequency (RF) electromagnetic wave of sufficiently high power density and tuned to match a natural E- or F-region plasma frequency, ionospheric magnetoionic wave modes may be excited and may generate RF electromagnetic sideband waves via nonlinear interactions. These secondary emissions, which may then escape from the ionosphere, have been termed stimulated electromagnetic emission or SEE. The frequency spectra of this radiation has been studied extensively, and a number of characteristic spectral features have been identified and in some cases related to particular plasma processes. The separation in frequency between the RF pump and the harmonics of the local electron gyrofrequency is critical in determining the amount of anomalous absorption suffered by the pump wave and the spectral properties of the stimulated sidebands. The pump can excite electrostatic waves which do not propagate away but can in some cases be observed via radio-wave scattering from the electron density fluctuations associated with them. These enhanced density fluctuations are created by processes commonly referred to as upper-hybrid and Langmuir turbulence. Langmuir turbulence has been the subject of 930-MHz scattering observations with antenna scanning through several pre-selected angles between the geographic and geomagnetic zenith directions, and a preference for pointing angles between the Spitze angle and geomagnetic field-aligned was identified. Other phenomena, such as the generation of enhanced electron temperatures and artificial aurora, have more recently been shown to have special behavior at similar angles, near but apparently not quite at field-aligned. In view of this evidence for angular structure
\end{abstract}

Correspondence to: B. Isham

(bisham@bc.inter.edu) in several pump-induced effects, in light of the rich variety of SEE phenomena strongly dependent on the geomagnetic field via the frequency interval between the pump and the gyrofrequency harmonics, and in view of the not yet understood but complex relationship between electrostatic fluctuations and SEE, it is of interest to investigate experimentally whether a similar angular structure is present in the various spectral features of the SEE signals and to compare the results with radar and other observations of RF-pump-induced effects. To this end we describe a simple two-element radio interferometer designed to search for aspect angle dependence of SEE features. We present an example of the initial data produced by this system, and draw preliminary conclusions based on the example data.

Key words. Ionosphere (Active Experiments) - Radio Science (Nonlinear Phenomena) - Space Plasma Physics (Turbulence) - Radio Science (Instruments and techniques)

\section{Introduction and summary of recent aspect angle re- sults}

Until relatively recently, the study of the angular variation of the phenomena observed during high-power RF ionospheric modification experiments has been largely ignored. Coherent radar aspect angle variation and scintillation of satellite and radio source signals have been used in the past to study artificially-created field-aligned irregularities at a number of locations around the world (Frolov, 2003), but work on the aspect angle variation of electron temperature enhancements, Langmuir turbulence, RF-induced optical emissions, and stimulated electromagnetic emissions (SEE) has only relatively recently begun (Isham et al., 1999a, c; Kosch et al., 
2000; Mishin et al., 2001; Pedersen et al., 2003; Rietveld et al., 2003; Frolov and Nedzvedski, 2004; Kosch et al., 2004; Frolov et al., submitted, 2004 ${ }^{1}$ ). The electron temperature and Langmuir turbulence studies have used the European Incoherent Scatter (EISCAT) Scientific Association high frequency (HF) transmitter and 930-MHz ultra high frequency (UHF) and 224-MHz very high frequency (VHF) incoherent scatter radars (ISRs) located at Ramfjordmoen near Troms $\varnothing$, Norway; the optical emission measurements have used both the EISCAT Troms $\varnothing$ facilities and the High-frequency Auroral Active Research Program (HAARP) HF transmitter and observatory near Gakona, Alaska, USA, and the SEE results come from the Sura HF facility near Vasil'sursk, Russia. The EISCAT HF facility (69.59 N latitude, 19.23 E longitude) has two low-gain antenna arrays, each capable of transmitting frequency-dependent effective radiated powers (ERPs) of about 150 to $330 \mathrm{MW}$ into the ionosphere at frequencies from 3.8 to $5.7 \mathrm{MHz}$ and from 5.3 to $8.0 \mathrm{MHz}$, respectively, and a high-gain array which can transmit about 600 to $1260 \mathrm{MW}$ ERP between 5.3 and $8.0 \mathrm{MHz}$, respectively. The HAARP facility (62.39 N latitude, $145.15 \mathrm{~W}$ longitude) is currently capable of transmissions between 2.8 and $8.0 \mathrm{MHz}$ with an ERP ranging from about 11 to $170 \mathrm{MW}$, respectively (K. Groves, private communication, 2003). The Sura facility (56.13 N latitude, $46.10 \mathrm{E}$ longitude) transmits between 4.3 and $9.5 \mathrm{MHz}$ with a maximum frequency-dependent ERP of between 80 and $280 \mathrm{MW}$, respectively (V. Frolov and E. Sergeev, private communications, 2003). The coordinates listed for the various facilities are geographic, and the ERP values apply to both peak and average power levels. A brief summary of the specifications of several past and present HF facilities is given in chapter four of Belikovich et al. (2002); some of the numbers above have been slightly updated from the values given there.

In the aspect angle experiments discussed here it is the variation in look angle from the observer to the region of the ionosphere illuminated by the RF pump beam which is important. This variation may be achieved by changing the angle of observation and, if necessary, in order to illuminate the region being observed, the angle of the pump beam. Typical pump beamwidths are on the order of ten degrees, so that adjusting the pump beam angle is not always necessary. Narrow-beam instruments, such as incoherent scatter radars, which typically have beams on the order of one degree wide, may be scanned within the region illuminated by the pump. Wider-beam instruments, such as imaging cameras, often cover all angles illuminated by the pump without any adjustments in pointing.

By varying the look angle we are exploring the effect of the magnetic field geometry on the physics of the pump-plasma interaction region. Geometrical parameters important in ionospheric turbulence experiments include

\footnotetext{
${ }^{1}$ Frolov, V. L., Nedzvetski, D. I., and Komrakov, G. P.: Peculiarities of SEE excitation using an oblique propagating HF powerful wave for ionosphere pumping, Izv. VUZov Radiofizika (in Russian), (Radiophys. Quant. Elec.), submitted, 2004.
}

look angle, relative altitude within the pump-plasma interaction region, pump wavelength, and pump polarization (Fig. 1). For incoherent scatter observations the radar $k$ vector is also important. In backscatter geometry, for example, the radar observes Langmuir and ion-acoustic waves having $k$ vector magnitudes equal to twice that of the probing radar. The Langmuir $k$ vector in turn determines an important reference height known as the radar matching height, defined as the altitude where twice the $k$ vector of the radar equals the $k$ vector of Langmuir waves which follow the Langmuir dispersion relation in the undisturbed background ionosphere and have a frequency equal to that of the RF pump. The matching height is thus the location where pump-enhanced Langmuir waves following the unperturbed dispersion relation may be observed by an incoherent scatter radar. Other important heights include the critical height, defined as the altitude where the frequency of the pump equals the local cold plasma frequency, and the upper-hybrid height, where the pump frequency equals the upper-hybrid frequency. The radar matching height lies below the critical height and will normally lie above the upper-hybrid height, but, depending on the radar $k$ vector and the electron temperature, it may also fall at or below the upper-hybrid level. The critical height is the maximum reflection height for O-mode waves; by this definition the maximum reflection heights for X-and Z-mode waves may be called the X-and Z-mode critical heights.

Important reference angles include geographic zenith (vertical), geomagnetic zenith (field-aligned), and the Spitze angle, which lies in between. The Spitze angle marks the end of the Spitze region, defined as the region in which an O-mode pump wave will reach the critical height before reflecting downward. Outside the Spitze region the pump wave refracts earthward before reaching the critical height. O-mode pump polarization is used in the majority of RF interaction experiments, as $\mathrm{X}$ mode reflects before reaching either the upper-hybrid or critical levels. An important property of the $\mathrm{O}$ mode is the possibility for it to couple into the $\mathrm{Z}$, or upper branch $X$, mode, and to thereby propagate through the $\mathrm{O}$-mode critical height. This can occur at angles close to the Spitze angle (Ellis, 1953, 1956). The pump wavelength determines the spacing between the high-power regions in the interference pattern formed below the critical height between the upward propagating pump wave and its reflection, known as the Airy pattern (Lundborg and Thidé, 1985, 1986). Finally, recent results suggest that under at least some circumstances the details of the pump beam pattern may be important. For example, Kosch et al. (2002b) report an observation in which a patch of stimulated optical emissions followed the motion of the pump beam rather than maintaining its position with respect to the geomagnetic field angle, as had been the case for previous results (Kosch et al., 2000). A unique feature of the more recent experiment was the use of the EISCAT high-gain, narrow-beam HF antenna, and this might, for example, indicate that the larger power density gradient at the edges of the beam has a role to play in the explanation of this result. 


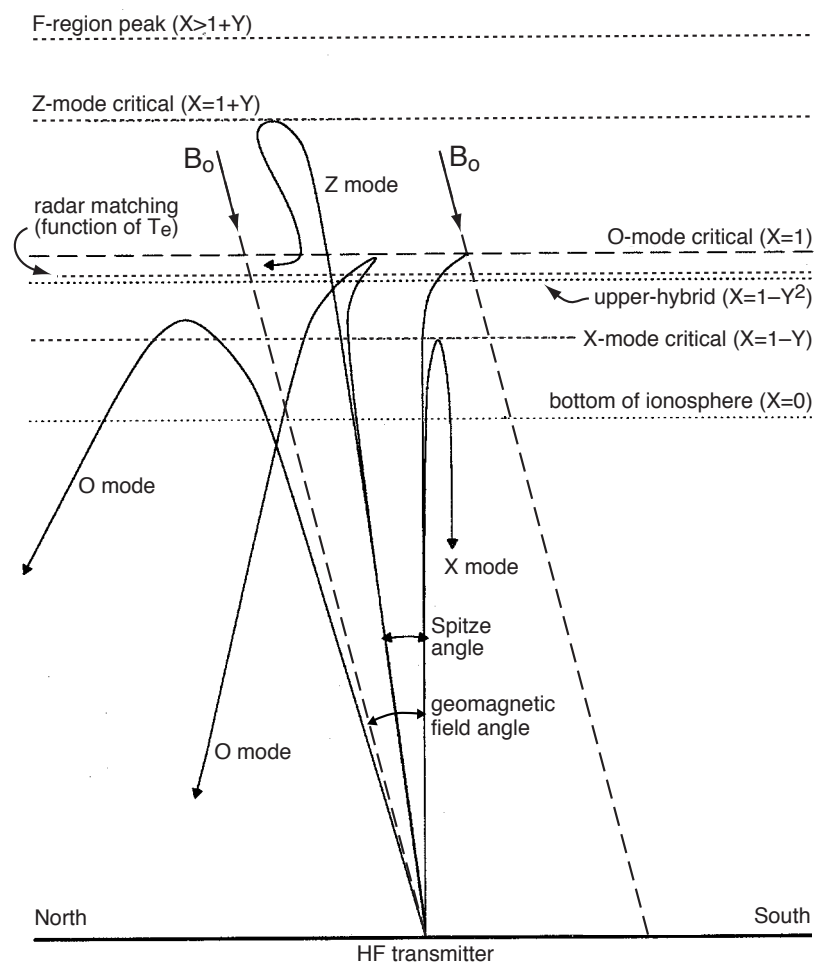

Fig. 1. Schematic view of HF ray paths in the bottomside F region for $f_{\mathrm{o}} \mathrm{F} 2>f_{\mathrm{hf}}+f_{\mathrm{ce}}$, where $f_{\mathrm{o}} \mathrm{F} 2$ is the peak F-region plasma frequency (the maximum O-mode reflection frequency), $f_{\mathrm{hf}}$ is the $\mathrm{HF}$, or RF, pump frequency, and $f_{\text {ce }}$ is the electron cyclotron, or gyro-, frequency. $\quad X=\left(f_{\mathrm{pe}} / f_{\mathrm{hf}}\right)^{2}$ and $Y=f_{\mathrm{ce}} / f_{\mathrm{hf}}$, where $f_{\mathrm{pe}}$ is the cold electron plasma frequency. The Spitze angle is equal to $\arcsin \left[(Y /(1+Y)]^{1 / 2} \sin \theta_{B}\right]$, where $\theta_{B}$ is the angle of the geomagnetic field and where both angles are with respect to geographic zenith, as shown in the figure. The radar matching height is given by $X=\left[1-\left(Y \sin \theta_{B}\right)^{2}\right] /\left[1+3\left(k \lambda_{\mathrm{D}}\right)^{2}\right]$, where $\lambda_{\mathrm{D}}=v_{\mathrm{e}} / 2 \pi f_{\mathrm{pe}}$ is the Debye length with $v_{\mathrm{e}}=\left(k_{\mathrm{B}} T_{\mathrm{e}} / m_{\mathrm{e}}\right)$ the electron thermal speed, $k_{\mathrm{B}}$ the Boltzmann constant, $T_{\mathrm{e}}$ electron temperature, and $m_{\mathrm{e}}$ electron mass. Adapted from Rietveld et al. (1993), Fig. 4.

\subsection{Electron temperature}

The increase in electron temperature caused by RF pumping is the phenomenon which gave the name "ionospheric heating" to this general class of space plasma experiment. Even so, observations of the variation in temperature with aspect angle to the geomagnetic field have only recently been made. Rietveld et al. (2003), on 7 October 1999, performed a threeposition radar scan using the EISCAT UHF radar. They observed at vertical, field-aligned, and half-way in between, the latter (middle) position corresponding to the local Spitze angle. The EISCAT HF transmitter was operated in O mode at $4.544 \mathrm{MHz}$ with an effective radiated power (ERP) of about $200 \mathrm{MW}$, with a 15-degree 3-dB full beamwidth and with the beam pointed at 0,6 , and 12-degree zenith angles for a complete radar angle scan, with a repeating cycle of 8-min on and 4-min off. The greatest temperature enhancements were normally seen at the field-aligned position, although due to

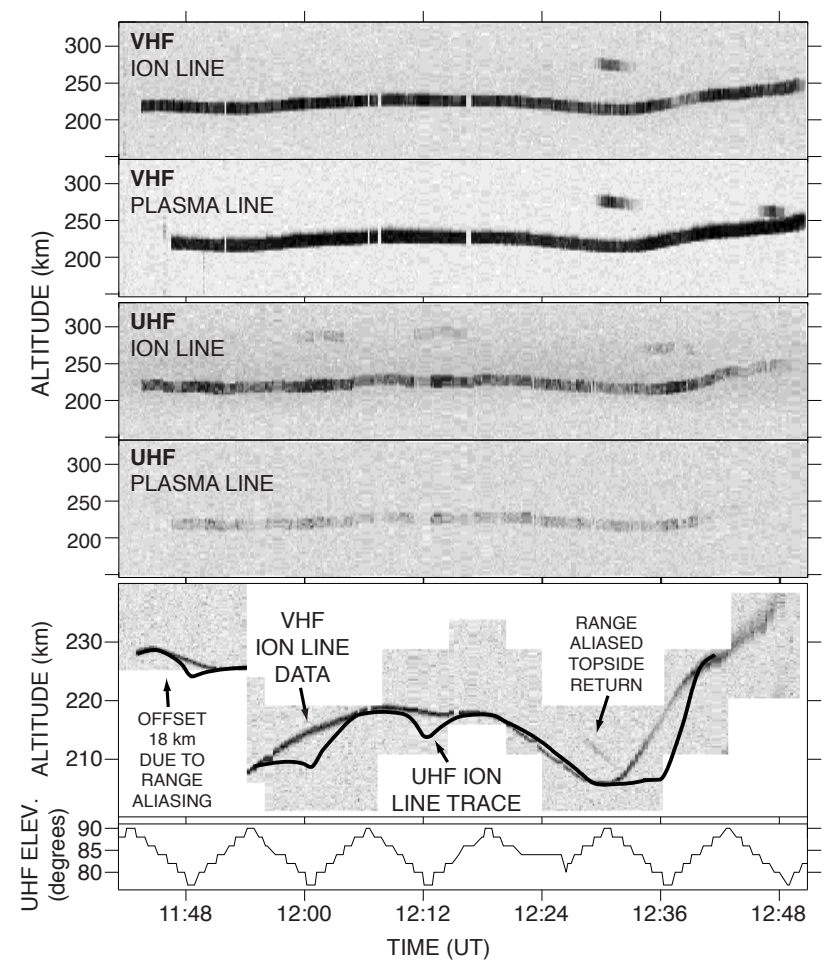

Fig. 2. Bottom- and topside ion and plasma line enhancements observed at EISCAT. Adapted from Isham et al. (1999c), Figs. 1 and 2.

the coarse resolution, it cannot be certain that field-aligned is the position of the true physical maximum. The behavior of the electron temperature for angles south of field-aligned has not been studied.

\subsection{Langmuir turbulence}

RF-pump-induced Langmuir turbulence occurs in the ionosphere when the electric field of the pump wave drives electrons to oscillate nonlinearly along the direction of the geomagnetic field. This turbulence may be seen by an incoherent scatter radar as enhanced scatter from ion-acoustic and Langmuir modes.

Figure 2 shows examples of topside ion line enhancements observed on 26 November 1997 with the EISCAT UHF and VHF radars (Isham et al., 1999c). In this case the pump frequency was $4.544 \mathrm{MHz}$, the transmitted power was 160 MW ERP pointed at 6-degree zenith angle (near the Spitze angle) with 15-degree pump beamwidth, and the transmitter cycle was 0.2 -s on and 9.8-s off. This observation was motivated by an experiment in August 1986 in which topside enhanced ion lines were observed with the EISCAT UHF radar (Isham et al., 1990). In that experiment the pump was operated mostly at $4.04 \mathrm{MHz}, 250 \mathrm{MW}$ ERP, normally pointed at 13-degree zenith angle (near geomagnetic fieldaligned) with 15-degree 3-dB full beamwidth, and was on for 20,30 , or 40 s every minute. The angle-sensitive $\mathrm{Z}$ mode was suggested as a means of propagation to the topside (Seliga, 1985; Mjølhus, 1990), and it was subsequently proposed 

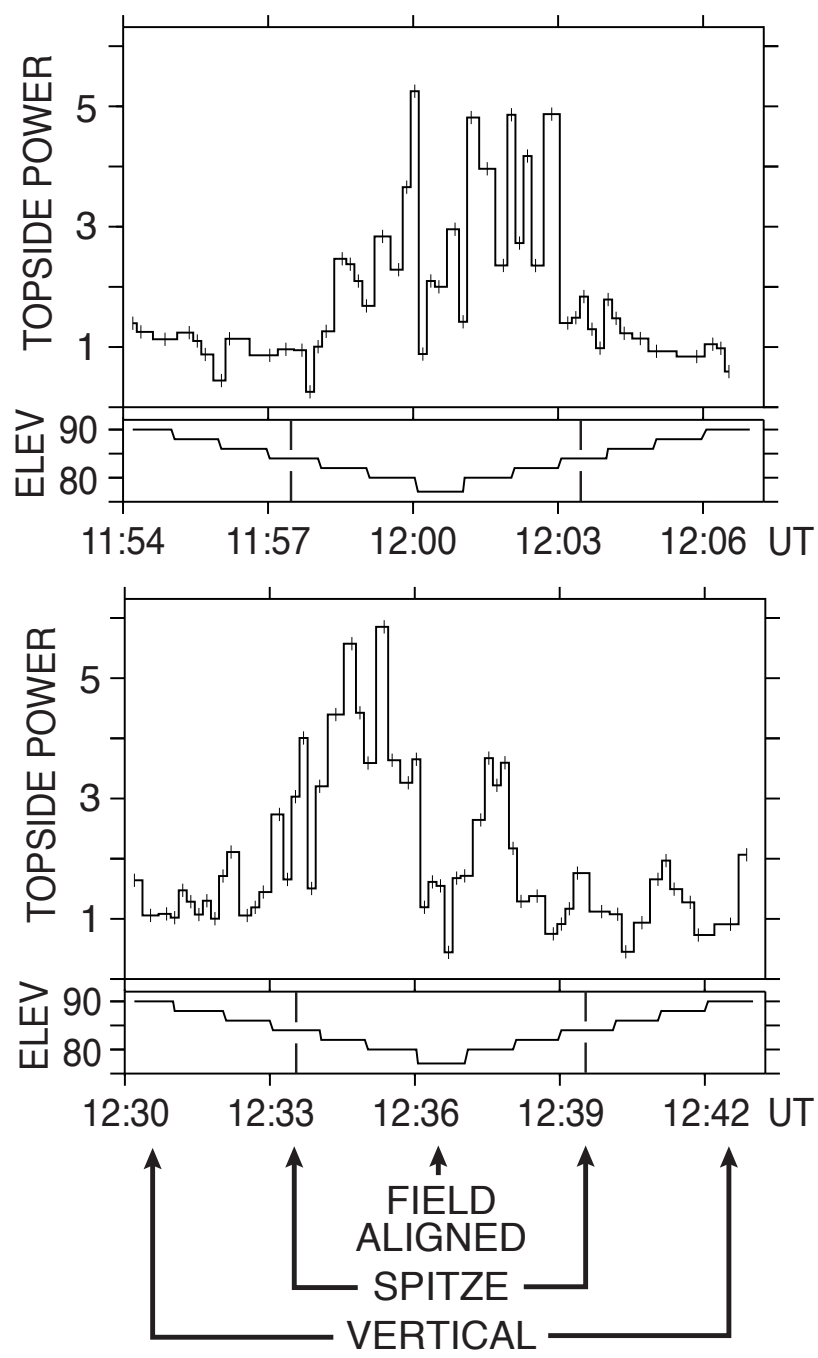

Fig. 3. Topside enhanced ion line power versus elevation angle for two antenna scanning cycles using the EISCAT UHF radar. Reproduced from Mishin et al. (2001), Fig. 2.

to study the angle dependence of the topside enhancement, and thus of the coupling from the bottomside, using antenna scanning. Somewhat surprisingly, as can be seen in Fig. 3, it was found that the maximum topside backscattered power occurs neither at the Spitze angle nor at field-aligned, but somewhere in between (Mishin et al., 2001). In these measurements the antenna did not scan north of the vertical or south of the geomagnetic field-aligned positions.

Figure 4 shows plasma line spectra measured on 1 December 1997 at $225 \mathrm{~km}$ range in the bottomside ionosphere during antenna scanning. The pump operation parameters were the same as on 26 November 1997. The intensities are greatest near the field-aligned position of the antenna and nearly vanish at vertical. There are two panels in the figure, chirped and unchirped. In the unchirped case an unmodulated $128-\mu \mathrm{s}$ pulse was used, while in the chirped case an identical pulse was transmitted, except that the transmission frequency was varied at a temporal rate of $-4.5 \mathrm{kHz} / \mu \mathrm{s}$, which translates

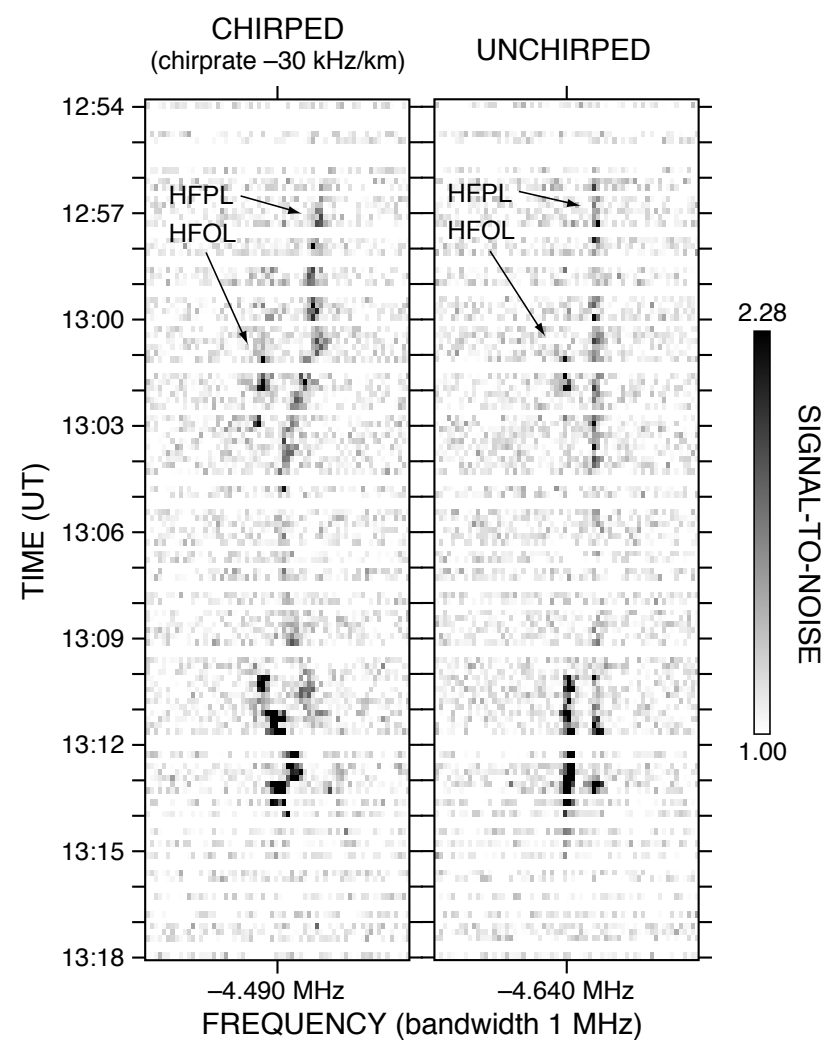

Fig. 4. UHF incoherent scatter radar spectra of HF-enhanced plasma lines from 1 December 1997. The spectra are most intense in the vicinity of the field-aligned position of the antenna, which in this example occurred at 13:00 to 13:01 and 13:12 to 13:13 UT. In both panels the right-hand line is the normal HF-enhanced plasma line, while the left-hand line is the free mode or outshifted line, shifted away from the radar transmission frequency, towards more negative frequencies for these downshifted waves. This effect was measured at HF pump frequencies of $4.9128 \mathrm{MHz}, 4.544 \mathrm{MHz}$ (the data shown here), and 4.040 MHz. Reproduced from Isham et al. (1999c), Fig. 3.

in the ionosphere to a geometrical rate of $-30 \mathrm{kHz} / \mathrm{km}$. The frequency scale of the chirped channel is thus linked to range. During scanning, features at a fixed altitude will vary in range and therefore also in frequency in the chirped spectrum. This variation of frequency with scan angle is apparent in the figure. Reversing this logic, we find that the larger frequency separation between the outshifted and normal RF-enhanced lines in the chirped vs. unchirped channels indicates that the volume of origin of the outshifted line lies slightly below that of the normal RF-enhanced line. The range resolution, $333 \mathrm{~m}$ in this case, is determined by the frequency resolution $(10 \mathrm{kHz})$ divided by the chirp rate $(30 \mathrm{kHz} / \mathrm{km})$ (Isham et al., 1999b).

Based on Fig. 4 it is possible to say that the bottomside plasma line enhancement is strongest near field-aligned, but it is not possible to say whether this maximum occurs exactly at field-aligned or perhaps some degrees offset from it, as is true for the topside ion line enhancements. Also, as the scan 


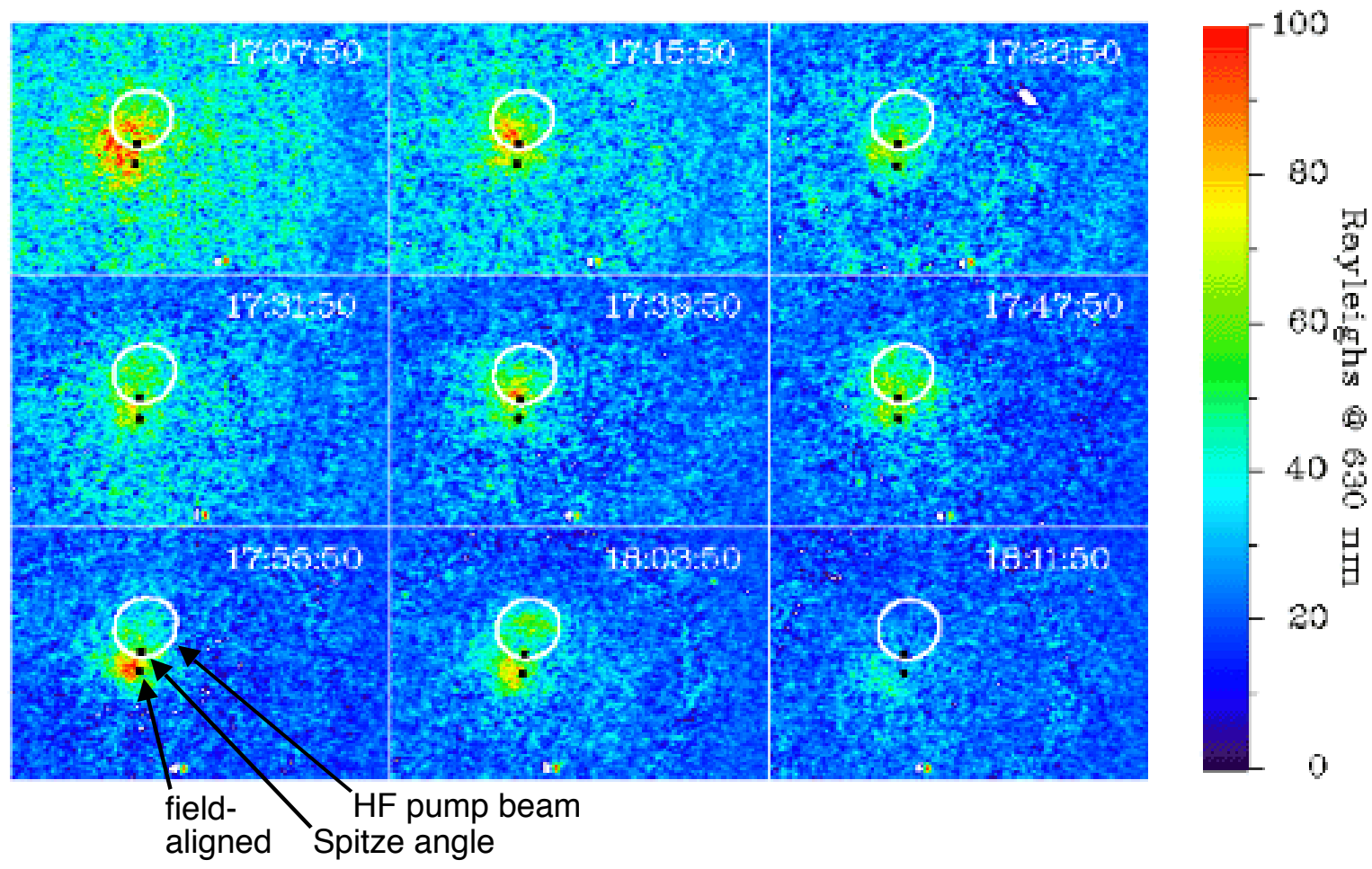

Fig. 5. Airglow at $630.0 \mathrm{~nm}$ between the Spitze and geomagnetic field-aligned positions. These positions are indicated for $230-\mathrm{km}$ altitude by the black squares; the circle indicates the half-power contour of the pump beam, which was centered on the zenith. Adapted from Kosch et al. (2000), Fig. 2.

was performed only to the field-aligned position, it is unknown whether the true maximum, or perhaps even a second maximum, might occur south of field-aligned. In September 2002 EISCAT UHF ion line observations were made at angles of about 6 and $12^{\circ}$ north and south of field-aligned and at field-aligned during 2-min on, 2-min off, O-mode pumping at $7.1 \mathrm{MHz}$ (between the fifth and sixth gyroharmonics). The results show strong enhanced returns at $6^{\circ}$ south of fieldaligned (Dhillon and Robinson, 2005). This is consistent with EISCAT UHF ion line data from November 2001, in which the antenna was scanned to $15^{\circ}$ south (about $2^{\circ}$ past field-aligned) during O-mode pumping at $5.423 \mathrm{MHz}$ (near the fourth gyroharmonic). The data indicate that there is a second ion line enhancement maximum on the bottomside south of field-aligned. Simultaneous optical observations suggest that these maxima may be two points on a ring of enhancements circling the field line (Kosch et al., 2004). Optical measurements are discussed in more detail below.

\subsection{Optical emissions}

The peculiar role played by the field-aligned direction is also apparent in observations made on 21 February 1999 of O(1D) 630.0-nm and on 3 February 2002 of O(1D) 630.0-nm and $\mathrm{O}(1 \mathrm{~S})$ 557.7-nm RF-induced optical emissions, at EISCAT and HAARP, respectively (Kosch et al., 2000; Pedersen et al., 2003). This type of pump-induced emission is also known as artificial airglow or artificial aurora. Figure 5 shows such observations, together with the half-power contour of the pump beam (assuming no refraction) and the intersections of the field-aligned and Spitze directions starting from, and at an altitude of $230 \mathrm{~km}$ above, the EISCAT HF transmitter. The HF transmitter was operated in O-mode at $4.04 \mathrm{MHz}$ with an effective radiated power (ERP) of about $70 \mathrm{MW}$, vertical pointing with 15 -degree $3-\mathrm{dB}$ full beamwidth, and with a repeating cycle of 4-min on and 4-min off. The Spitze angle was computed using the 4.04-MHz pump frequency. The data show that the region of artificial emissions is shifted away from the beam center and toward the field-aligned direction in a fashion similar to the shift of the pump-induced Langmuir waves observed by the EISCAT UHF radar, as previously described. The HAARP experiment, which produced similar results, used $\mathrm{O}$ mode at transmission frequencies of 4.8 and $5.8 \mathrm{MHz}$ with continuous full power (about 60 and 90 MW ERP, respectively), with a 20-point pump beam azimuth scan at 15-degree zenith angle, and with a dwell time of $30 \mathrm{~s}$ at each point in the scan.

Subsequent observations at EISCAT on 12 November 2001 at $5.423 \mathrm{MHz}$, near the fourth gyroharmonic, revealed a ring structure in pump-enhanced emissions at $630.0 \mathrm{~nm}$, $557.7 \mathrm{~nm}$, and in white light, which after about two minutes collapsed into the filled circle typical of all previous observations (Jussila, 2002; Kosch et al., 2002b, 2004). Enhanced emission from $\mathrm{N}_{2}^{+}$at $427.8 \mathrm{~nm}$ was also detected, although 
not imaged (Holma et al., submitted, 2004²). The ERP was $550 \mathrm{MW}$ with a 7.5-degree 3 -dB full beamwidth, pump beam scanning between zenith angles of $3^{\circ}$ north to $15^{\circ}$ south, and a repeating transmission cycle of 2-min on, 2-min off. The ring structure points to a geometrical effect, but equally significant is the fact that the structure was not fixed at a particular angle but remained centered in the pump beam for all of the imaged wavelengths while the beam was scanned. A major difference between the November 2001 observations and other high-latitude observations is that the pump ERP is roughly five times greater, and this may allow the geometrical properties of the pump beam, in particular the magnitude and gradient of the pump electric field, to dominant over the geometry of the magnetized ionospheric plasma.

The excitation of $630.0-\mathrm{nm}$ emissions requires a source of energetic electrons having energies above $1.96 \mathrm{eV}$. After accounting for electron energy loss processes, the effective $\mathrm{O}\left({ }^{1} \mathrm{D}\right)$ excitation energy in the $\mathrm{F}$ region becomes approximately $3 \mathrm{eV}$; for $557.7-\mathrm{nm} \mathrm{O}\left({ }^{1} \mathrm{~S}\right)$ emissions the corresponding values are $4.17 \mathrm{eV}$ and approximately $6 \mathrm{eV}$ (Bernhardt et al., 1989). Excitation of $\mathrm{N}_{2}^{+}$first negative 427.8-nm emissions requires electron energies of $19.8 \mathrm{eV}$, assuming no losses (Stolarski et al., 1967). RF pump-enhanced optical emissions from neutral $\mathrm{N}_{2}$, in the $\mathrm{N}_{2}$ first positive band at $660 \mathrm{~nm}$ and requiring electron energies of $7.3 \mathrm{eV}$, assuming no losses (Stolarski et al., 1967), have been reported in the E region above Arecibo, Puerto Rico, using a pump frequency of $3.175 \mathrm{MHz}$ (Djuth et al., 1999). At Arecibo the geomagnetic field has a zenith angle of about $45^{\circ}$, a significantly different geometry than in the higher-latitude locations where the scanning observations discussed here have been carried out (Isham et al., 2000). Evidence for the existence of energetic electrons accelerated during RF pumping is also provided by Arecibo incoherent scatter radar (ISR) observations of RF-enhanced natural plasma lines (Carlson et al., 1982; Gurevich et al., 2000).

Pump-induced plasma turbulence holds promise as a source of energetic electrons. Such turbulence can consist of either Langmuir and ion-acoustic waves (Langmuir turbulence) or upper and lower hybrid waves (upper-hybrid turbulence). Previous studies, based on SEE, anomalous absorption, and electron temperature measurements, indicate that there is a minimum in the level of upper-hybrid excitation at the gyroharmonic frequencies (Stubbe et al., 1994; Robinson et al., 1996; Frolov et al., 1998, 2001; Mjølhus, 1998). More recently, both airglow and coherent backscatter radar signals have been shown to vanish when the pump frequency is tuned to the third gyroharmonic (Kosch et al., 2002a), supporting the role of upper-hybrid turbulence as the source of the energetic electrons. However, measurements near the fourth gyroharmonic show strong pump-induced airglow, suggesting, in the absence of upper-hybrid turbulence, that Langmuir turbulence must have been the mechanism responsible

\footnotetext{
${ }^{2}$ Holma, H., Kaila, K. U., Kosch, M. J., and Rietveld, M. T.: Recognizing the blue emission in artificial aurora, Adv. Space Res., submitted, 2004.
}

for accelerating the electrons (Kosch et al., 2004). Whether both of these conclusions are valid, or if they apply under different conditions, has not yet been determined.

\subsection{Stimulated electromagnetic emission}

Stimulated radiofrequency electromagnetic emission, or SEE, is a rich and complex phenomenon with intimate connections to striations and turbulent processes (Leyser, 2001). The geomagnetic field plays a dominant role in SEE, and the morphology of the SEE spectrum depends dramatically on the proximity of the pump frequency to a harmonic of the local electron gyrofrequency (Frolov et al., 2001). These properties of SEE strongly suggest that features in the SEE spectrum must in all probability also depend on geomagnetic aspect angle.

The importance of the gyroharmonic frequencies was emphasized by Stubbe et al. (1994), who divided SEE features into two general classifications: gyrofeatures, which appear only when the pump is in the vicinity of an electron gyroharmonic frequency, and universal features, which may appear at most or all ionospheric pump frequencies. In general, the detailed morphology of any given SEE feature will change as the pump frequency is varied (Frolov et al., 2001). Distinctions may also be made between steady-state and transient features, and between features seen under unpreconditioned as opposed to preconditioned pumping conditions. Most reported results are for the steady state, which results from pumping at a high enough average pump power to create and sustain small-scale artificial field-aligned irregularities. In particular, small-scale irregularities, which form near the upper-hybrid level, are known to be intimately associated with SEE, and, under these circumstances, upper-hybrid turbulence is thought to be important in the SEE generation process. When irregularities remain from a previous pump-on period and are present at the start of a new pump turn-on, the situation is known as preconditioned. For unpreconditioned observations, before artificial irregularities have developed, and also near the gyroharmonics, where upper-hybrid turbulence and artificial irregularities are known to be suppressed, Langmuir turbulence is suspected to be dominant. This is thought to be the case for the fast narrow continuum (FNC) (Leyser, 2001) and the first component of the broad upshifted maximum $\left(B_{1} M_{1}\right)$ (Frolov et al., 1998), respectively.

A schematic overview of the behavior of many of the major steady-state features in the SEE spectrum as a function of pump frequency relative to the fourth and higher gyroharmonic frequencies is shown in Fig. 6. Steady-state features which may be seen primarily or only at the third gyroharmonic include the downshifted peak (DP) and the broad symmetrical structure (BSS), respectively (Stubbe and Kopka, 1990), whose spectral variations in the vicinity of the third harmonic are shown in Figs. 7 and 8. The variation of the downshifted maximum (DM), the upshifted maximum (UM), and the broad upshifted maxima 1 and $2\left(\mathrm{BUM}_{1}\right.$ and $\left.\mathrm{BUM}_{2}\right)$ near the fourth gyroharmonic, are shown in Fig. 9. Note the wide variation in frequency offsets of the various spectral 
features, and that the emissions may be downshifted, upshifted, or both simultaneously, relative to the RF pump frequency.

Although many theoretical explanations have been advanced, the generation of SEE is still not well-understood. It is likely that the various spectral features have different origins, and that these differences have an influence on the direction of arrival of the associated waves.

To date, most SEE measurements have been carried out at locations nearly directly under the RF interaction region, and no thorough studies of SEE versus angle of arrival have been reported (Leyser, 2001). Carozzi et al. (2001) measured the polarization state of SEE and concluded that some spectral components have oblique incidence, suggesting the possibility of separate source regions for different components. Gurevich et al. (2002), quoting V. Frolov (private communication), report that a significant intensification of the SEE downshifted maximum was observed at a pump frequency of $5.6 \mathrm{MHz}$ when the pump beam was turned from vertical to magnetic field-aligned. Frolov et al. (submitted, 2004 ${ }^{1}$ ) discuss measurements of the SEE broad upshifted maximum made while the direction of the RF pump beam was scanned in the geomagnetic field plane between +16 and $-16^{\circ}$ from vertical.

\section{Technique}

SEE was discovered and has most often been measured using a spectrum analyzer connected to an HF receiving antenna (Thidé et al., 1982; Stubbe et al., 1984). Although spectrum analyzers provide only moderate (several-second) time resolution, they have the large, $120-\mathrm{dB}$ or greater dynamic range sometimes required to detect the weak SEE signals lying near the ionospherically-reflected (and possibly ground-path-propagated) pump wave, which may have 100-dB-greater power than the SEE sidebands.

On the other hand, modern digital data-taking systems provide the best possible time resolution. In addition, these systems can be designed to record raw voltage samples, a feature which allows for the possibility of multi-channel interferometry. However, the dynamic range of digital systems is limited by the number of bits available in their analog-to-digital converters (ADCs). Current digital systems typically use 14bit ADCs, which provide $78 \mathrm{~dB}$ of dynamic range; additional dynamic range may be obtained through the use of a filter to notch out the pump frequency. Alternatively, more bits may be sampled. At least one 23-bit system is in use for SEE observations, providing $132 \mathrm{~dB}$ of dynamic range (Carozzi et al., 2002). Such systems are ideal, but expensive.

A coherent four-channel 14-bit digital receiver has been constructed by the Polar Geophysical Institute in Murmansk, Russia. The receiver frequency is tunable between 1.5 and $32 \mathrm{MHz}$, with a $1.875-\mathrm{MHz}$ complex sampling frequency and a $350-\mathrm{kHz}$ hardware-limited data bandwidth. Unintegrated SEE spectra with $915.5-\mathrm{Hz}$ frequency resolution and $1.082-\mathrm{ms}$ time resolution, for example, may be achieved

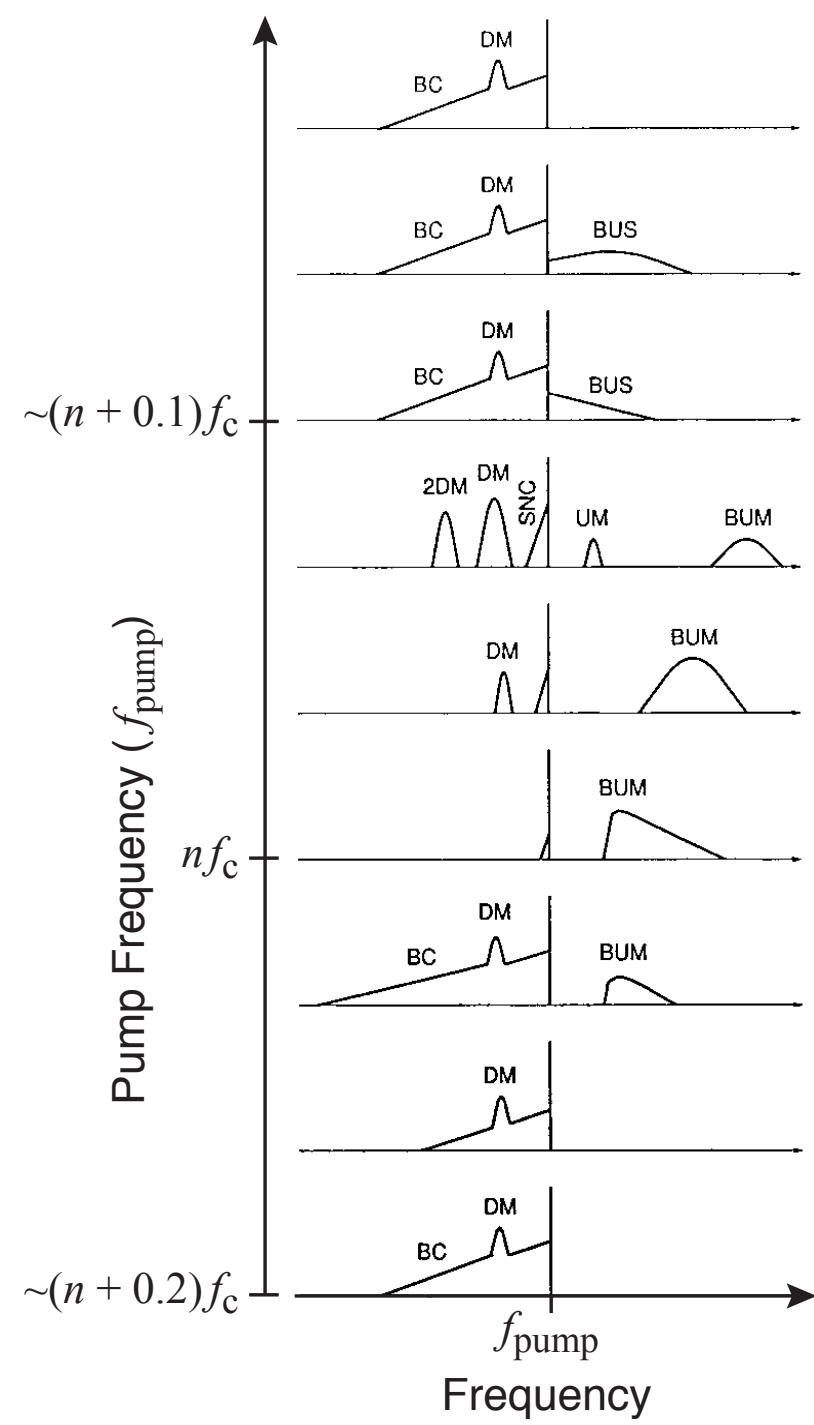

Fig. 6. Schematic depicting many of the major steady-state SEE features and their variation as a function of pump frequency $f_{\text {pump }}$ relative to the gyroharmonic frequencies, $n f_{\mathrm{c}}$, for $\mathrm{n}>3$, where $f_{\mathrm{c}}$ is the electron gyro-, or cyclotron, frequency. The pump frequency is different in each panel, as indicated on the vertical axis; however, the horizontal frequency scales of the individual panels have been shifted so that the different pump frequencies are aligned, as indicated on the bottom horizontal axis. In this way the differences in the nature of the SEE spectra as a function of offset from the pump can be illustrated as a function of changing pump frequency relative to the fourth and higher gyroharmonic frequencies. The features shown on this diagram are the broad continuum (BC), downshifted maximum (DM), broad upshifted structure (BUS), downshifted maximum first cascade (2DM), slow narrow continuum (SNC), upshifted maximum (UM), and broad upshifted maximum (BUM); note that the BUM marked in this figure is the BUM $_{2}$ (Frolov et al., 1998). Adapted from Leyser (2001), Fig. 32.

by processing 2048-point complex voltage data vectors. The system is designed to be expanded from four to eight channels. 

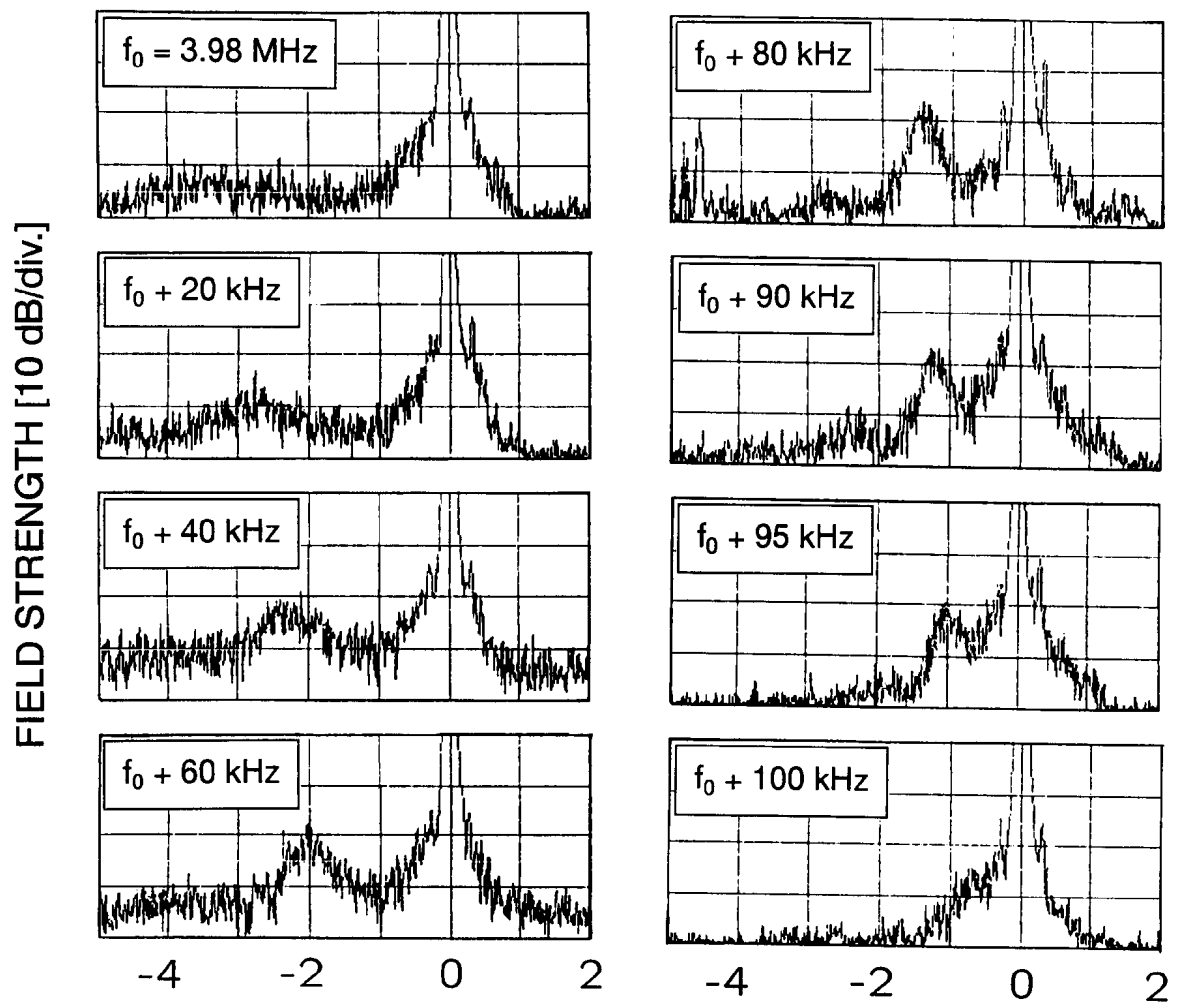

$\Delta \mathrm{f}[\mathrm{kHz}]$

Fig. 7. The behavior of the downshifted peak (DP) near the third gyroharmonic. Note that the frequency scale is just a few kHz wide. These data were taken using the Troms $\varnothing$ HF facility in November 1989. Reproduced from Stubbe and Hagfors (1997), Fig. 15.

For the simplest interferometric observations of coherence and cross phase, two channels may be connected to two simple spaced HF electric dipole antennas. If four dipoles are available, a measure of the polarization dependence of the results may be included by arranging antennas in pairs of orthogonal polarizations at the two locations and connecting them to four receiver channels. Alternatively, three antennas could be used to measure angular variation on two baselines, for example, north-south and east-west, thus determining a unique point of origin of the signal in the sky.

The above procedures all use the phase of the cross spectrum between antenna pairs to find the signal-weighted average position of the SEE source region in the plane of the antenna baseline. Since the interferometer pair will be looking at signals originating in the region of the ionosphere illuminated by the pump beam, the angle between interferometric fringes, that is, the angle between position angles with path length differences of exactly one wavelength, must be greater than the full beamwidth of the HF pump antenna. For example, the EISCAT low-gain HF antenna arrays have a full beamwidth of $15^{\circ}$ at the $3-\mathrm{dB}$ points, so that a twoelement SEE antenna spacing which results in interference fringes greater than $15^{\circ}$ apart is required for the proposed measurements. However, since RF-induced phenomena may occur at pump power levels far below $-3 \mathrm{~dB}$ relative to the maximum effective radiated power density of the pump antenna pattern, the distance between fringes should in practice be several times greater than indicated by the $3-\mathrm{dB}$ points of the pump beam pattern alone.

A useful guide to a practical maximum in the fringe spacing is the width of the beam pattern of the individual elements in an interferometer pair. For example, for a center frequency of $5.468 \mathrm{MHz}$ and free-space wavelength of $54.86 \mathrm{~m}$, commercially-available 27.43 -m-long folded fullwave dipoles might be used. Assuming such a dipole is mounted horizontally approximately $4.5 \mathrm{~m}$ above the ground, the dipole pattern will have $-3-\mathrm{dB}$ points at zenith angles of about $\pm 30^{\circ}$ along the dipole axis and $\pm 45^{\circ}$ perpendicular to it. Within the band of commonly available RF pump frequencies (roughly 3 to $10 \mathrm{MHz}$ ) the dipole pattern changes very little; in addition, these figures depend very little on the exact mounting height above the ground.

Based on the above example, we might desire $100^{\circ}$ between fringes, a little more than half the sky. To achieve this, the spacing of the antenna pairs should be on the order of one pump wavelength. For example, 50-m antenna spacing would provide a geometrical fringe spacing of about $100^{\circ}$ at $4 \mathrm{MHz}$ and about $50^{\circ}$ at $8 \mathrm{MHz}$. As discussed in the Introduction, variations in RF-induced phenomena have previously been measured with angular resolutions of about $3^{\circ}$, 


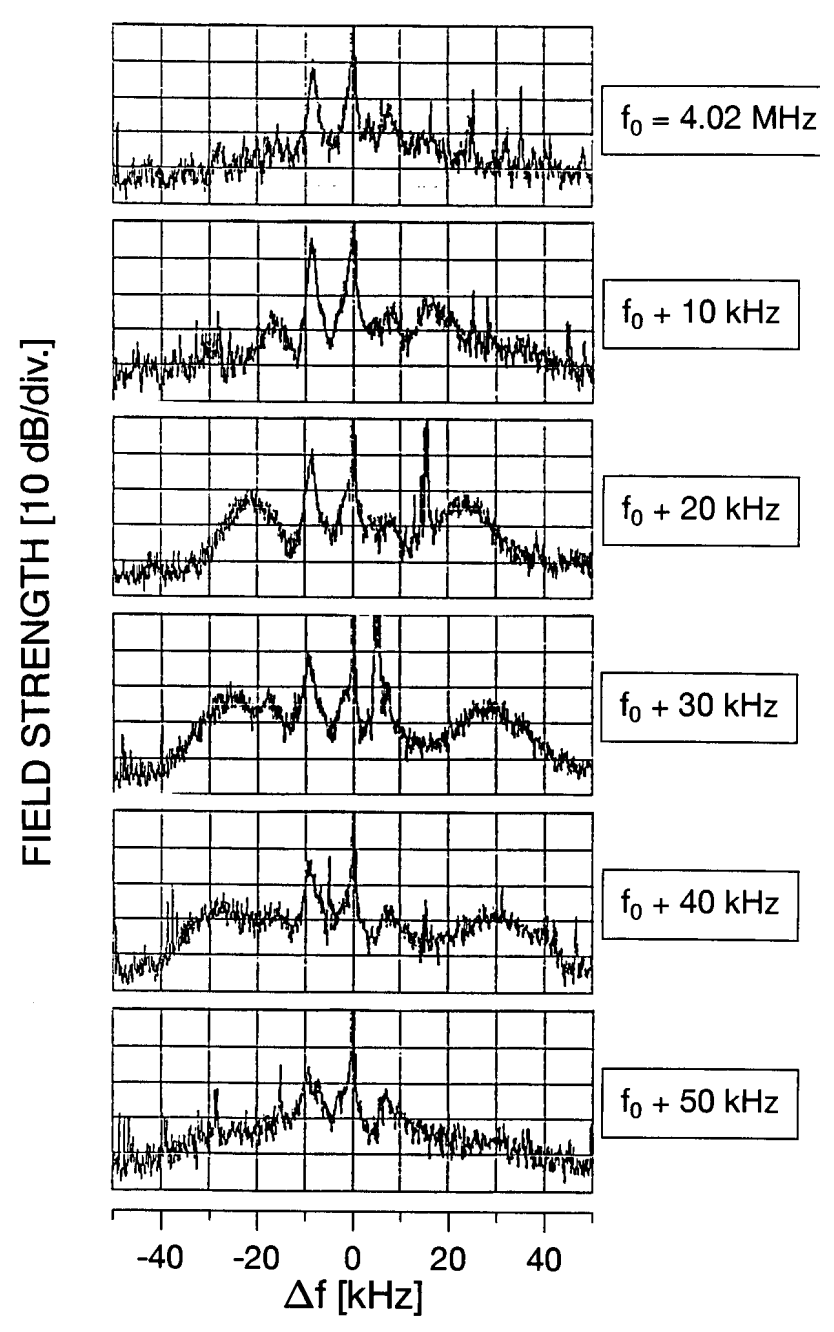

Fig. 8. The behavior of the broad symmetrical structure (BSS) near the third gyroharmonic. These data were taken using the Troms $\varnothing$ HF facility on 9 November 1989, 15:02 to 15:58 UT. Reproduced from Stubbe and Hagfors (1997), Fig. 16.

and for improved measurements we might desire 1-degree resolution. At $100^{\circ}$ fringe spacing, this would then correspond to 100 points across the sky, or, since there are $360^{\circ}$ of cross phase between fringes, for 1-degree spatial resolution we should have a phase resolution of one point per $3.6^{\circ}$ of interferometric cross phase. For the steady-state SEE observations discussed here a time resolution of ten seconds would be acceptable, which, for the example above with $1.082-\mathrm{ms}$ time resolution, would allow nearly ten thousand integrations per phase measurement. Given the typically good signalto-noise characteristics of SEE spectra, it is expected that 100 points across the phase spectrum may easily be resolved.

In the past, interferometry between antenna pairs has been carried out by looking for variations in the strengths of the sums and differences of the signals from two antennas while scanning an interference null across the region of interest. In our case scanning of the interference pattern might be done by adjusting the phase angle between the two sample streams

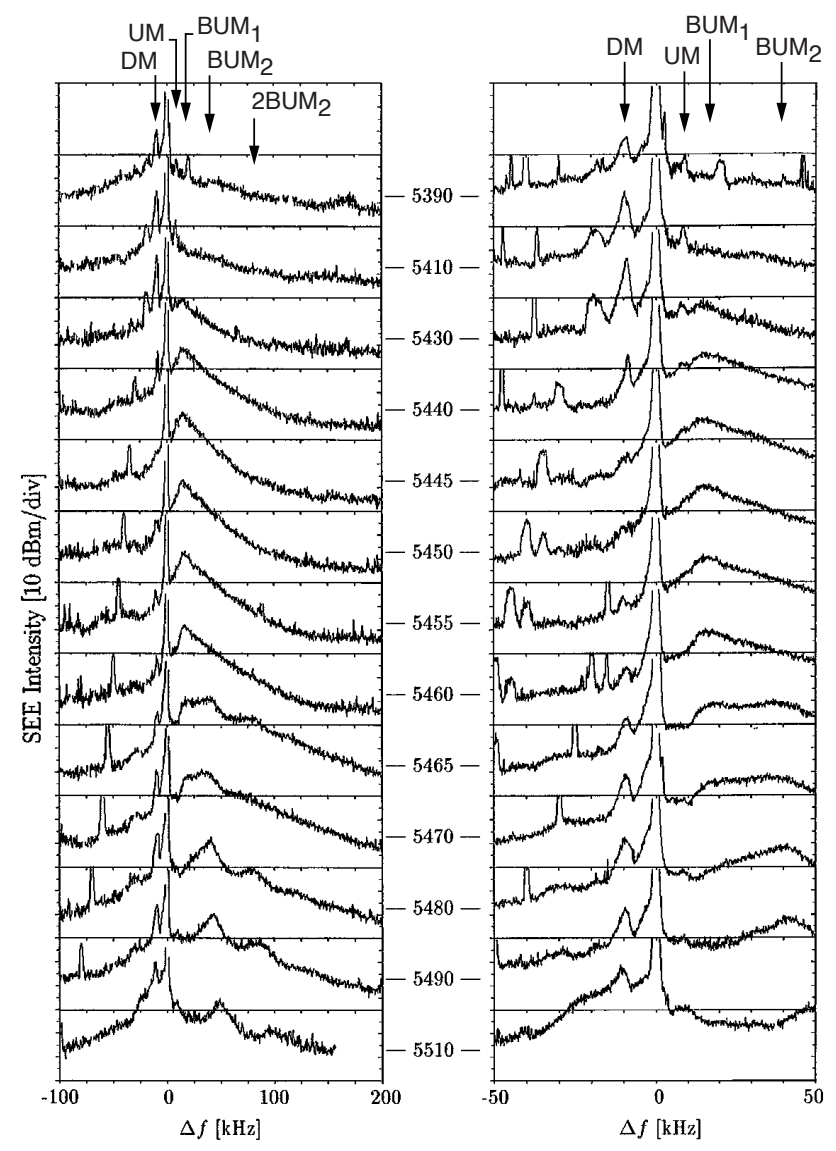

Fig. 9. The behavior of the downshifted maximum (DM), upshifted maximum (UM), the broad upshifted maxima 1 and $2\left(\mathrm{BUM}_{1}\right.$ and $\left.\mathrm{BUM}_{2}\right)$, and the second harmonic of the $\mathrm{BUM}_{2}\left(2 \mathrm{BUM}_{2}\right)$ near the fourth gyroharmonic. These data were taken at the Sura HF facility on 3 September 1997, 13:10 to 13:50 LT, using an effective radiated power of $150 \mathrm{MW}$. Two spectra with different frequency spans are presented for each pump frequency. Adapted from Frolov et al. (1998), Fig. 1.

in order to place the null at any desired position. This technique depends sensitively on having identical gains in the two channels, which can also be adjusted by applying calibration adjustments to the sample streams. However, with increases in available computing power, the cross-correlation technique, which has the advantage of having an algorithm which can process all the available data in one operation, has become more practical.

As discussed above, for the simplest interferometric observations, the required spacing of the antenna pairs will be on the order of one pump wavelength. More complicated antenna geometries designed for radio imaging could make use of many dipoles arranged on multiple-wavelength baselines and sampled on individual raw data channels. This arrangement, given a suitable distribution of baselines, would remove the signal-weighted average-position limitation of the cross-phase technique. 


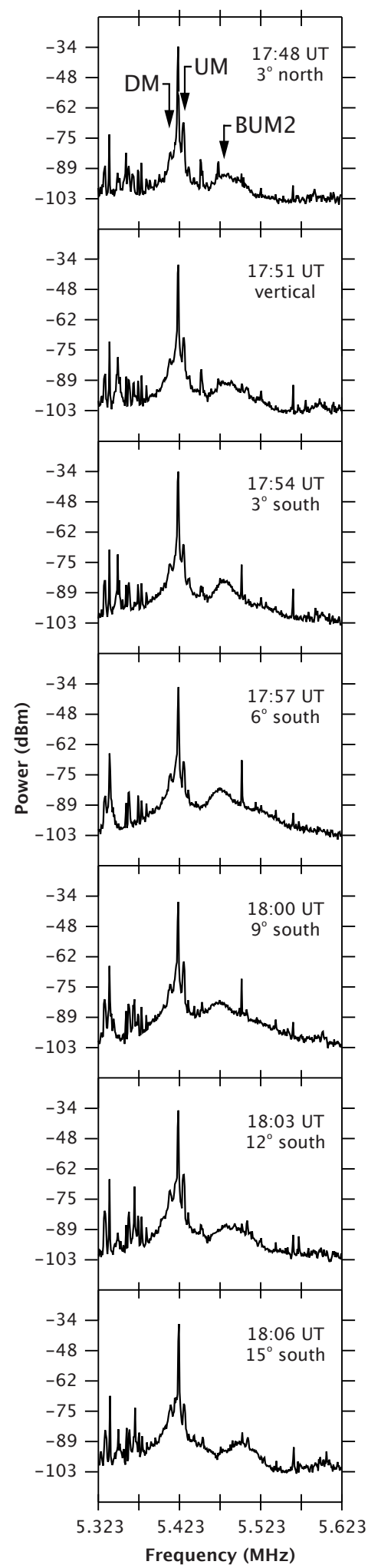

Fig. 10. SEE data during aspect angle scanning of the RF pump beam along the magnetic meridian. Time is in UT (local time minus one hour). The pump frequency is $5.473 \mathrm{MHz}$, near the fourth gyroharmonic. The SEE spectra have been integrated for $1 \mathrm{~min} 45 \mathrm{~s}$ during the 2-min pump-on period.

\section{Observations}

An early indication of the possible dependence of SEE on aspect angle can be seen in low time resolution SEE data taken at EISCAT on 12 November 2001, using a single dipole antenna connected to a spectrum analyzer system during a period in which the beam of the EISCAT HF facility was scanned in 3-degree steps between $15^{\circ}$ south and $3^{\circ}$ north (see Fig. 10). The pump was operated at $5.423 \mathrm{MHz}$ at about $550 \mathrm{MW}$ ERP with 7.5-degree 3-dB full beamwidth, with a transmitter cycle of 2-min on, 1-min off. The data show the $\mathrm{BUM}_{2}$ to be especially peaked between 3 and $9^{\circ}$ south, with the closest approach of the $\mathrm{BUM}_{2}$ to the pump frequency occurring at about $9^{\circ}$ south. As previously noted, ISR and optical data measured near Troms $\emptyset$ show special behavior near $9^{\circ}$ south, also not directly along the geomagnetic field.

However, this result is inconclusive as there is only one example, and as changes in the ionosphere might produce the same effect. For example, the pump frequency was near the fourth gyroharmonic, and if the ionosphere were to drift near or through the local gyroharmonic frequency at just the time when the pump beam was moving from 9 to 6 to $3^{\circ}$ south, then a similar result might be seen (see Figs. 9 and 6). Incoherent scatter and ionosonde data show that the critical height corresponding to $5.423 \mathrm{MHz}$ was changing near the time when this SEE data was taken, but they do not have adequate resolution to determine how the relation to the gyroharmonic might have changed during the period of the antenna scan. On the other hand, the downshifted and upshifted maxima (DM and UM) also appear in the spectra, for all pointing angles. Although the DM appears weak in some of the plots, the UM is still present, and in past observations the DM has never failed to be present when the UM is present (Leyser, 2001). This indicates that the gyroharmonic was not reached during the period shown in Fig. 10. Furthermore, the $\mathrm{BUM}_{2}$ feature appears about $50 \mathrm{kHz}$ from the pump frequency, distinguishing it from the $\mathrm{BUM}_{1}$ and indicating that the pump frequency lies approximately $50 \mathrm{kHz}$ above the gyroharmonic (Leyser et al., 1989; Stubbe and Kopka, 1990; Frolov et al., 1998) (see Fig. 9).

The SEE interferometer system described here was first operated at the Sura HF facility during March 2004. Unfortunately, SEE signals were relatively weak and the results to date are inconclusive. A second campaign was carried out during late September and early October 2004, using the EISCAT HF facility at Ramfjordmoen near Troms $\varnothing$, Norway, and strong SEE signals were seen. During that campaign the SEE system was located at Ramfjordmoen $15 \mathrm{~km}$ away from the HF pump transmitter, and voltages were normally recorded from three antennas. The antennas were three $27.43-\mathrm{m}$-long folded full-wave dipoles mounted as discussed in the example in the previous section, that is, horizontally approximately $4.5 \mathrm{~m}$ above the ground with the antenna axes aligned in the east-west direction, with baselines of $50.2 \mathrm{~m}$ east-west and $55.6 \mathrm{~m}$ north-south. An identical fourth antenna provided an alternative north-south baseline of $106.2 \mathrm{~m}$, and this was sometimes used. As 

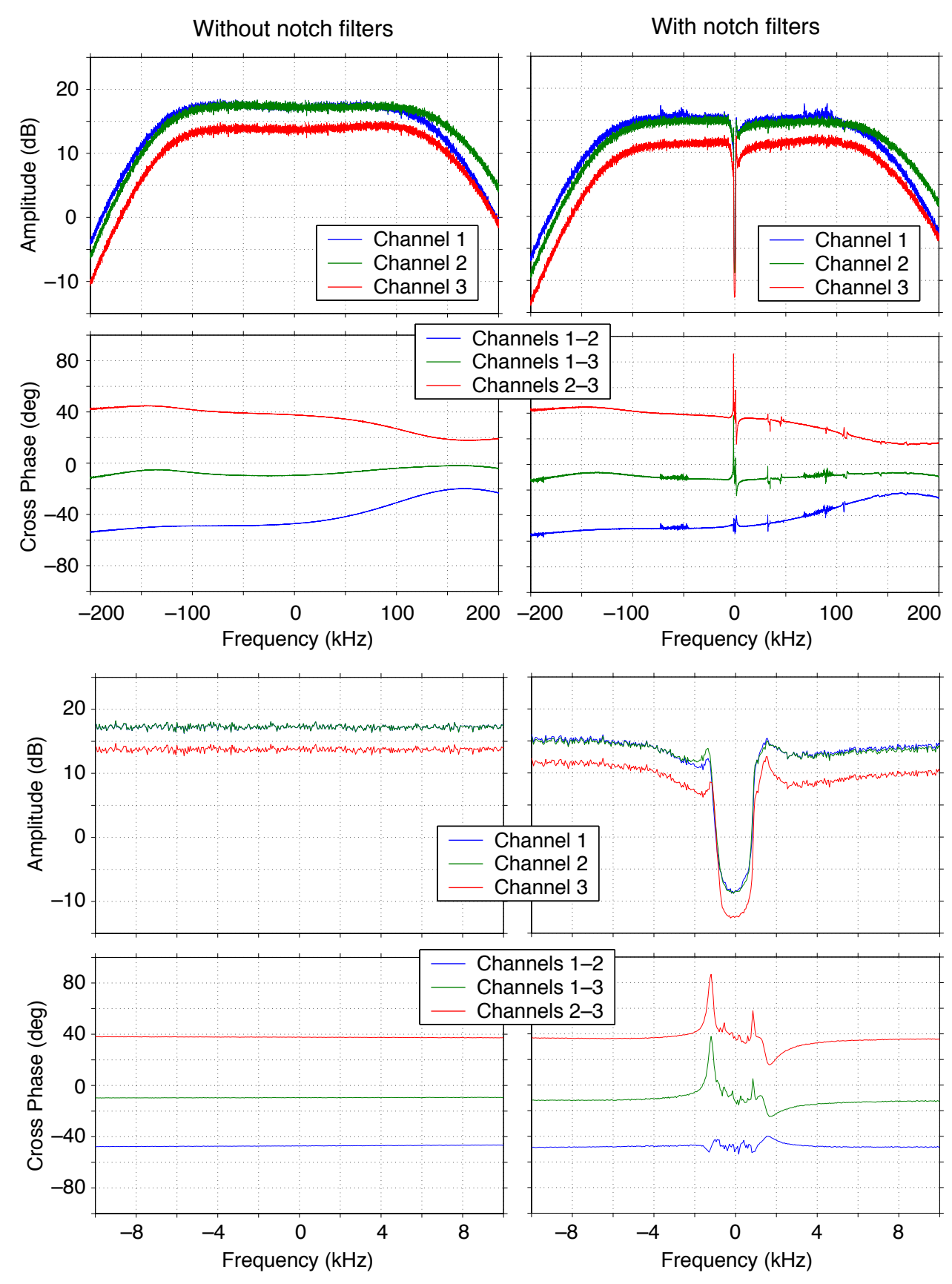

Fig. 11. The magnitude and cross-phase responses of the three digital receiver channels used to record the interferometric data channels, with and without the 5.423-MHz notch filters. For these measurements a white noise source was divided into the three receiver channels and data was recorded simultaneously in all three. Because of this, the fluctuations in the different channels are nearly identical, and the blue trace (channel 1) is, for example, nearly perfectly hidden behind the green trace (channel 2) in the lower left panel. The red trace (channel 3 ) is about $3.5 \mathrm{~dB}$ below the other two due to differences in the signal paths. The filters have 3 - $\mathrm{dB}$ points at about $\pm 1 \mathrm{kHz}$ from the center frequency. The frequency resolution in these plots is $50 \mathrm{~Hz}$. Note that the filters were not used in recording the data presented in this report.

discussed above, 50-m baselines provide a geometrical fringe spacing of about $50^{\circ}$ at $8 \mathrm{MHz}$ and about $100^{\circ}$ at $4 \mathrm{MHz}$; 1-degree angular resolution would then require resolving 50 or 100 points across the 360 -degree phase spectrum.

During the campaign, in order to gain experience with the limitations in the dynamic range provided by the 14-bit ADCs, data were recorded both with and without the use of notch filters. These notch filters are currently available at 4.040, 4.544, 4.9128, 5.423, and $5.752 \mathrm{MHz}$; all but the last correspond to frequencies allocated to the EISCAT HF transmitter, all but the first may be used at the Sura HF facility. An example of the notch filter frequency response is given in Fig. 11. 

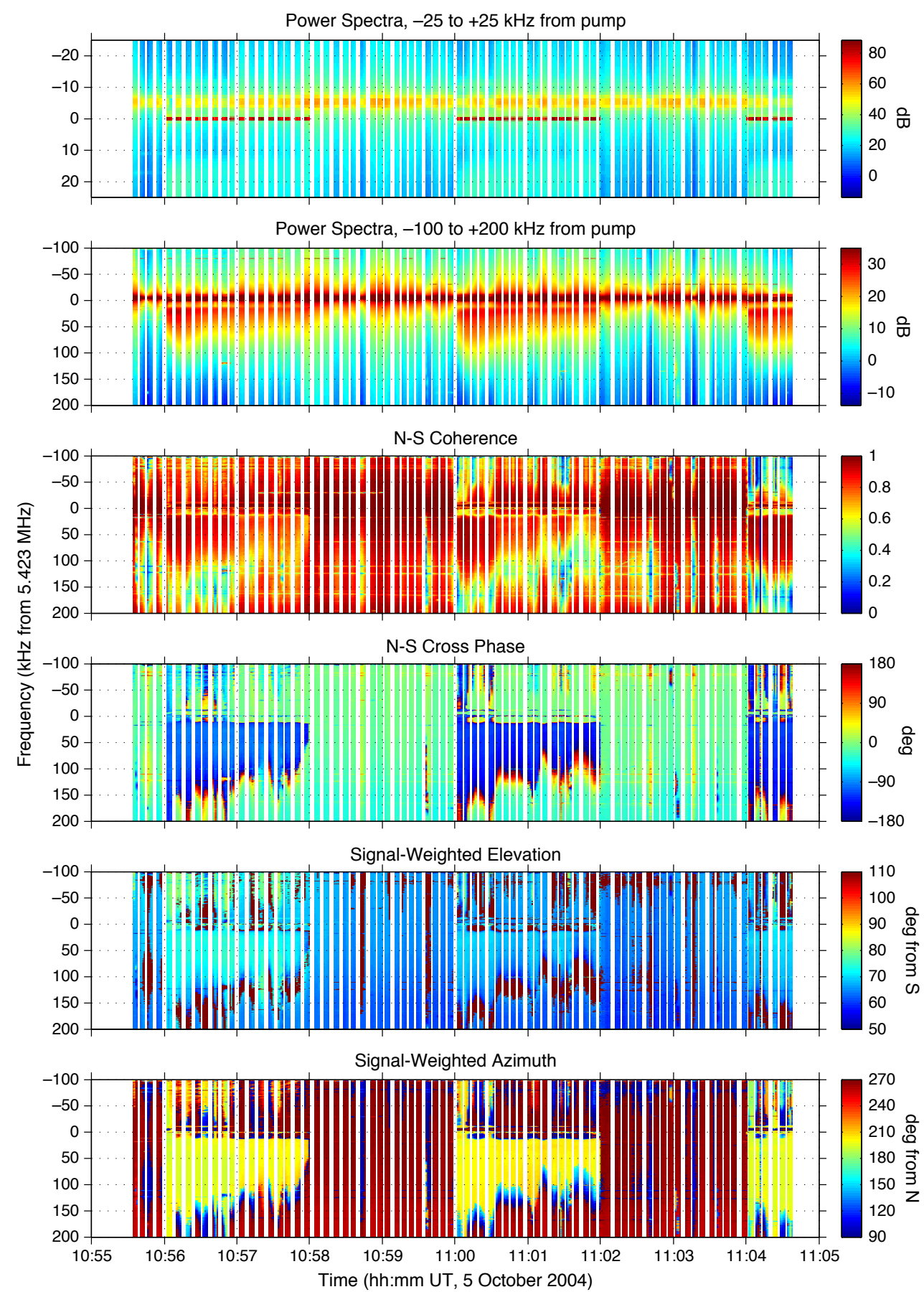

Fig. 12. SEE interferometer data from 5 October 2004 at EISCAT on Ramfjordmoen. The top panel shows the power spectrum of the central antenna on a $\pm 25 \mathrm{kHz}$ frequency scale. The pump can be clearly seen cycling 2-min on, 2-min off; the strong feature at about $6 \mathrm{kHz}$ is interference. Due to the high levels of the pump and the interference, no SEE features can be seen in this panel. The second panel shows the SEE power spectrum from -100 to $+200 \mathrm{kHz}$ on a more limited intensity scale. The $\mathrm{BUM}_{1}$ can be clearly seen, but the DM, which is present, is obscured by the interfering signal. Panels three and four show the coherence and cross phase along the north-south path, and the bottom two panels show the elevation and azimuth angles computed from the cross phase of the north-south and east-west antenna pairs. Azimuth is measured from geographic north and ranges from 90 (due east) through 180 (geographic south) to 270 (due west), while elevation ranges from 0 (on the southern horizon) through 90 (vertical) to 180 (on the northern horizon), although only elevations from 50 to 110 are plotted. These scales were chosen in order to show any signals from north of vertical on a continuous scale with signals from the south. The data were recorded in 4-s-long files, with a 2- to 4-s data gap between files. The time and frequency resolutions in this figure are $1 \mathrm{~s}$ and $1 \mathrm{kHz}$, respectively. 
Data from the recent EISCAT campaign are still in the early stages of processing, but an example which illustrates the technique is presented in Fig. 12. At the time this data was recorded, the RF pump was operating in $\mathrm{O}$ mode at $5.423 \mathrm{MHz}$ in a repeating cycle of 2-min on, 2-min off. The pump beam width was approximately $7.5^{\circ}$ and the effective radiated power (ERP) was about $700 \mathrm{MW}$. During the first pump-on, at 10:56 UT, the HF beam was pointed at $14.0^{\circ}$ south of geographic zenith; during the following two, beginning at 11:00 UT and 11:04 UT, the pump was oriented $17.5^{\circ}$ south. These positions corresponded to elevation angles of 76 and $72.5^{\circ}$. The SEE receiving site was located approximately $15 \mathrm{~km}$ north of the EISCAT HF transmitter. No notch filters were used during the period shown in this example.

The top two panels of Fig. 12 show the SEE power spectrum vs. time, as recorded by the central antenna. The first panel shows the pump and a strong interfering signal; in the second panel the first component of the broad upshifted maximum $\left(\mathrm{BUM}_{1}\right)$ is clearly seen between about 15 and $60 \mathrm{kHz}$ above the pump frequency. The strong interfering signal obscures the downshifted maximum (DM), which is also present during this time period. This combination of features indicates that the pump frequency is about $5 \mathrm{kHz}$ above the local gyroharmonic (see Fig. 9). The third panel of Fig. 12 shows the coherence between the north and south antennas. There are many regions of high coherence, including regions of cosmic noise and of man-made interference, as well as the regions where the $\mathrm{BUM}_{1}$ feature is located. The fourth panel shows the cross phase, and clearly indicates regions of constant phase corresponding to the $\mathrm{BUM}_{1}$. The bottom two panels show the elevation and azimuth as computed from the cross phases of the north-south and east-west antenna pairs.

Further details of the BUM $\mathrm{B}_{1}$ are shown in Fig. 13, which has a more selective frequency scale. The elevation angle corresponding to the $\mathrm{BUM}_{1}$ is about $74^{\circ}$ during the pumping cycle at 10:56 UT and about $70^{\circ}$ during the cycles at 11:00 and 11:04 UT. It is particularly interesting that the variation in the angle of the SEE source region as seen by the interferometer reflects the variation in the direction of the pump beam, 76 and $72.5^{\circ}$, respectively, as described in the preceding paragraph. The azimuth angle is in the vicinity of $200^{\circ}$, not exactly north-south; variation in azimuth angle is also on the order of the change in pump beam direction, but does not appear to correlate with the pump-beam-swinging pattern.

It is interesting to compare the $\mathrm{BUM}_{1}$ in Fig. 13 with DM data, shown in Fig. 14. When coherence is high, the elevation angle for the DM appears to be about $77^{\circ}$ during the pumping cycle at 10:56 UT and about $73^{\circ}$ during the cycles at 11:00 and 11:04 UT, suggesting that the source region of the DM, too, is responding to the motion of the pump beam. However, the value of the elevation angle appears to be sensitive in some way to the magnitude of the coherence, and this remains to be investigated in detail. The azimuth angle of the DM, about $205^{\circ}$, is similar and arguably equal to that of the $\mathrm{BUM}_{1}$ within the apparent range of fluctuations in the measured values. As with the $\mathrm{BUM}_{1}$, the azimuth is not aligned north-south and varies within a range of several degrees but in no obviously discernable pattern.

The coherences of both the BUM 1 and DM appear to decrease, by 10 or 20 or greater percentages, during the course of the 2-min pump-on periods. This decrease in coherence is mainly gradual and apparently repeatable. The coherence of the DM is much more variable than that of the $\mathrm{BUM}_{1}$, and appears to have a dependence on the orientation of the antenna baseline.

The data used to produce these results were calibrated, using data such as that shown in Fig. 11, to correct for distortions in the amplitude and phase of the measurement system. However, these are preliminary data, and all possible factors which might affect the computed angles may not yet have been taken into consideration. Furthermore, the data examined so far are a small sample. These results should therefore not be taken as final, but as preliminary calculations which may need revising as more data is processed.

\section{Discussion}

As described above, RF-pump-enhanced incoherent scatter ion and plasma lines have been observed in the topside ionosphere above Tromsø (Isham et al., 1990, 1999a, c). The mechanism by which this occurred was ascribed by the observers to the conversion of the originally transmitted $\mathrm{O}$ mode pump wave into the $\mathrm{Z}$ mode, which may subsequently penetrate the ionosphere to either the Z-mode critical height or to the topside O-mode critical height (Ellis, 1953, 1956; Mjølhus and Flå, 1984; Seliga, 1985; Mjølhus, 1990). During this observation the F-region peak frequency $\left(f_{\mathrm{o}} \mathrm{F} 2\right)$ was less than the Z-mode critical frequency, so that Z-mode waves were able to reach the topside and might have excited instabilities at or near the topside critical level (where the RF pump frequency equals the topside local cold plasma frequency) and thereby created the observed enhanced ionacoustic and Langmuir waves. At least one other topside excitation mechanism has been suggested, namely the transmission of power through the F region via pump-modulated kinetic tunneling of electrons (Eliasson, 2001; Revenchuk et al., 2001).

In the Z-mode scenario, for a horizontally stratified medium one would expect the pump wave to penetrate the critical height at the Spitze angle and to be limited to a narrow angular window, making it likely that the topside plasma turbulence feature be of a limited lateral extent along the magnetic meridian plane. This was one of the original motivations for the ISR antenna scanning experiment. Figure 1 gives a sketch of the various possible propagation paths for the situation where the electron density is high enough for the $\mathrm{Z}$ mode to be reflected. The topside echo would then be absent, if the Z-mode explanation of the topside echoes is the correct one. An observation under these conditions could be used as a test for distinguishing between the $\mathrm{Z}$ mode and kinetic tunneling mechanisms. 

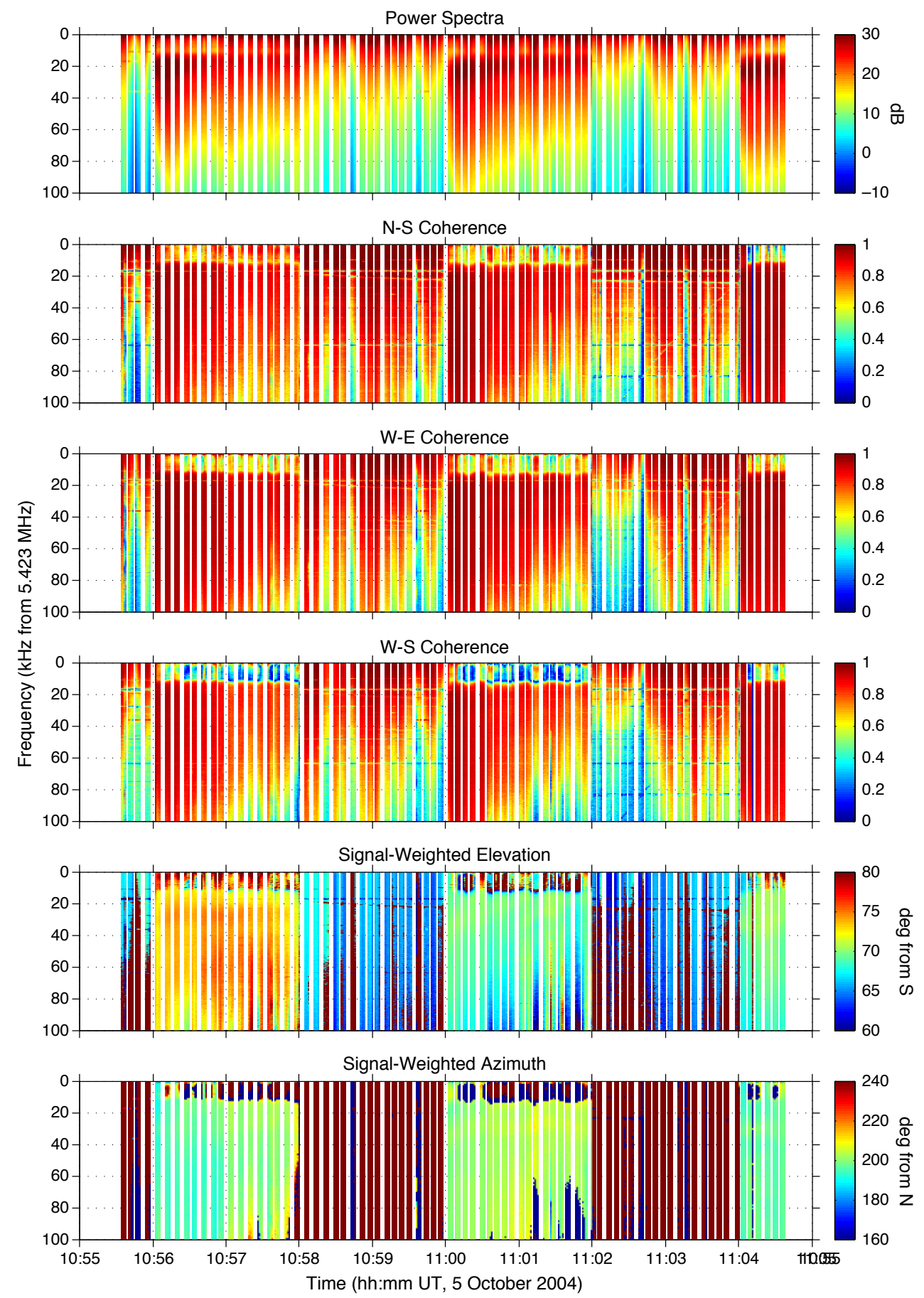

Fig. 13. SEE interferometer data from 5 October 2004 at EISCAT on Ramfjordmoen. This is the same time period as in Fig. 12 but with an expanded view of the frequency scale encompassing the $\mathrm{BUM}_{1}$, and showing the coherences for all three antenna baselines plus elevation and azimuth angles computed for the $\mathrm{BUM}_{1}$ source region. The elevation angle of the $\mathrm{BUM}_{1}$ is about $74^{\circ}$ during the pump cycle beginning at 10:56 UT and about $70^{\circ}$ during the follow two cycles. Time and frequency resolutions are $1 \mathrm{~s}$ and $1 \mathrm{kHz}$.

Langmuir turbulence may be subdivided into cascading and cavitating regimes (sometimes loosely referred to, respectively, as weak and strong turbulence). Both of these may be observed using incoherent scatter radars, cascading turbulence at the radar matching height and cavitating turbulence near the critical height (Robinson, 1997). For
Langmuir turbulence to be excited in a magnetized plasma, the pump must drive electrons along or closely aligned to the direction of the ambient magnetic field. At the critical level the pump electric field is parallel to the geomagnetic field and should therefore excite cavitating turbulence, which should be observable by both the EISCAT 224-MHz (VHF) 

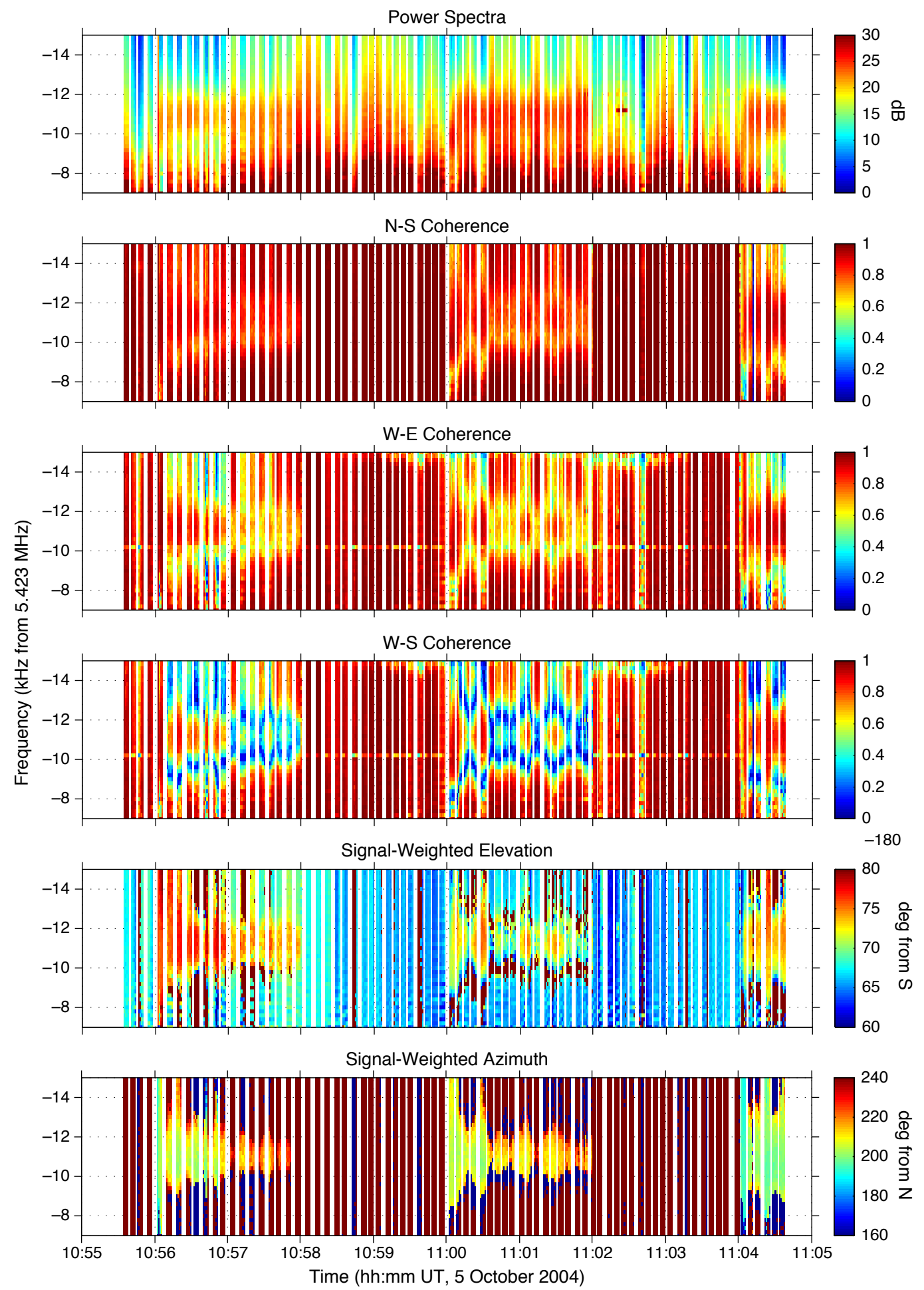

Fig. 14. SEE interferometer data from 5 October 2004 at EISCAT on Ramfjordmoen. This is the same time period as in Fig. 12 but with an expanded view of the portion of the frequency scale encompassing the DM, and showing the power spectrum, the coherences for all three antenna baselines, plus elevation and azimuth angles computed for the DM source region. The DM can be seen between about -10 and $-12 \mathrm{kHz}$ relative to the pump frequency. The elevation angle of the DM is about $77^{\circ}$ during the pump cycle beginning at 10:56 UT and about $73^{\circ}$ during the follow two cycles. Time and frequency resolutions are $1 \mathrm{~s}$ and $200 \mathrm{~Hz}$. 


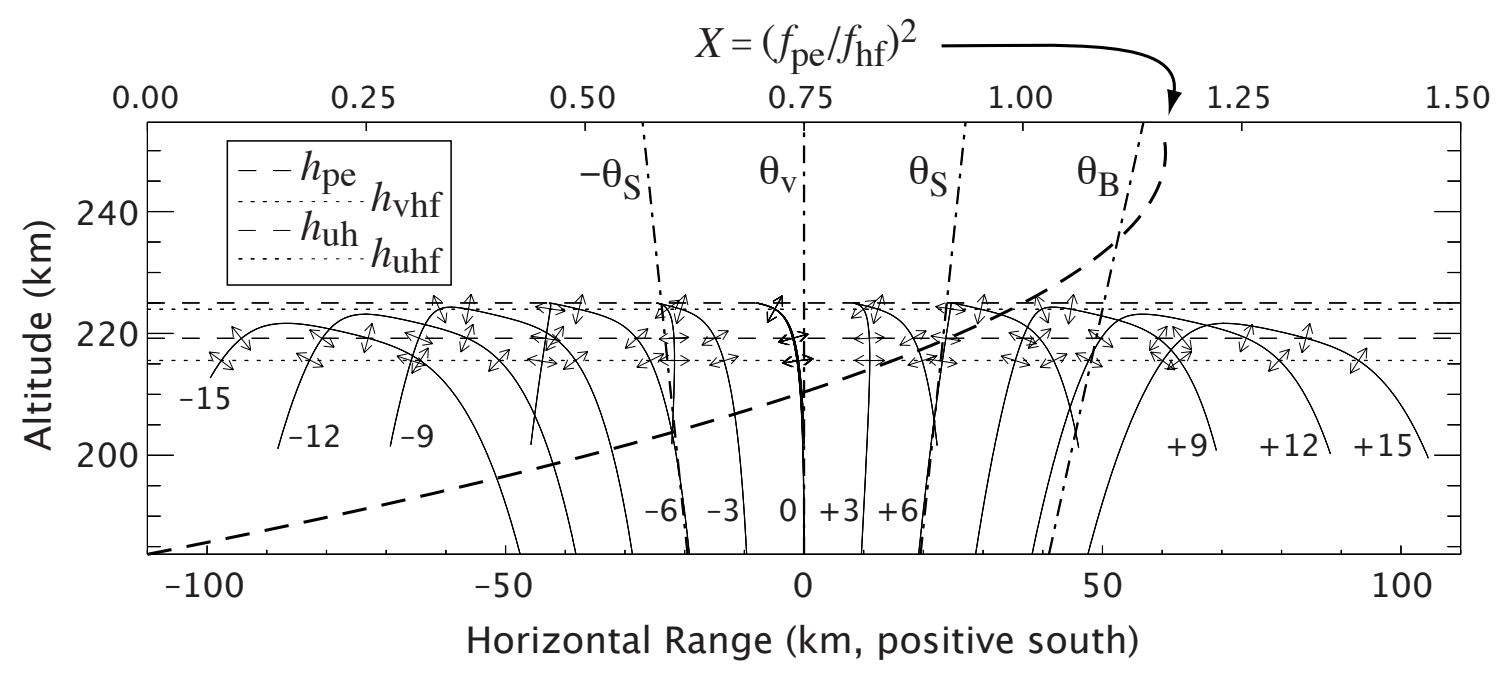

Fig. 15. Computed ray paths and electric field polarizations of an RF pump wave in a smooth ionosphere at the EISCAT VHF radar matching height $\left(h_{\mathrm{vhf}}\right)$, the upper-hybrid resonance height $\left(h_{\mathrm{uh}}\right)$, and the EISCAT UHF radar matching height $\left(h_{\mathrm{uhf}}\right)$. The ray paths are shown for angles between -15 and $+15^{\circ}$ from vertical $\left(\theta_{\mathrm{v}}=0^{\circ}\right)$ in the magnetic meridian plane at the EISCAT site near Troms $\varnothing$, Norway. The plasma density profile is given in units of $X=\left(f_{\mathrm{pe}} / f_{\mathrm{hf}}\right)^{2}$, where $f_{\mathrm{pe}}$ is the cold plasma frequency and $f_{\mathrm{hf}}$ is the HF, or RF, pump frequency. The plasma frequency gradient equals $30 \mathrm{kHz} / \mathrm{km}$ at the critical height $\left(h_{\mathrm{pe}}\right)$, here chosen to be $225 \mathrm{~km}$ for an O-mode pump frequency of 4.544 MHz. These parameters match those of the data shown in Figs. 2 and 4. A parabolic density profile was assumed, producing an $f_{\mathrm{o}} \mathrm{F} 2$ of $4.9 \mathrm{MHz}$ at $250 \mathrm{~km}$ altitude. The electron gyroharmonic frequency is $1.33 \mathrm{MHz}$ and the geomagnetic field angle $\left(\theta_{B}\right)$ is $12.9^{\circ}$ from vertical. The Spitze angle $\left(\theta_{\mathrm{S}}\right)$ is then $6.1^{\circ}$. The matching heights were computed assuming an electron temperature of $2000 \mathrm{~K}$. As the electron temperature decreases the matching heights rise; at an electron temperature of $1050 \mathrm{~K}$ the VHF matching height would lie about $30 \%$ closer to the critical level and the UHF matching height would coincide with the upper-hybrid resonance height.

and $930-\mathrm{MHz}$ (UHF) incoherent scatter radars. As shown in Fig. 15, in the case of the VHF radar, the pump electric field has a large component in the geomagnetic field direction at both the matching and critical heights. The excitation of both cascading and cavitating Langmuir turbulence should therefore be relatively easily achieved. In the case of the UHF radar, the matching height lies several $\mathrm{km}$ below the critical height at a point where the pump electric field is largely horizontally polarized, and the excitation of cascading turbulence should be more difficult. These calculations suggest that the maximum turbulence seen by the UHF radar ought to lie at the critical height between the Spitze angle and the vertical, yet the maximum occurs outside the Spitze, at an angle where the pump wave does not reach critical, and, perhaps even more puzzling, at an altitude several $\mathrm{km}$ below the maximum height reached by the pump. Mjølhus et al. (2003) consider this problem and are unable to suggest an explanation. At positions beyond the Spitze to the south, conditions for strong polarization of the pump electric field along the geomagnetic field are present when the pump wave is propagating downward after reflection. Whether this is important or not is an open question. Beyond the Spitze to the north (Mjølhus, 1990), field-aligned polarization occurs while the ray is still propagating upwards.

Based on these considerations there appears to be no convincing reason why the geomagnetic field-aligned direction should play such a prominent role, as is implied in the ISR observations of electron temperature and of enhanced ion and plasma lines, as shown in Figs. 2, 3, and 4, and in airglow observations, such as those shown in Fig. 5. Gurevich et al. (2002) have proposed an explanation for enhanced airglow at field-aligned based on HF self-focusing on pump-induced plasma density striations, but they do not discuss possible mechanisms for the temperature and turbulence maxima observed between the Spitze and field-aligned angles.

The above considerations focus mainly on Langmuir turbulence. However, it is well known that upper-hybrid turbulence, occurring at the upper-hybrid level several $\mathrm{km}$ below the critical height, is important for the production of plasma density striations and SEE. The SEE features, such as the downshifted maximum and broad continuum, may be signatures of cascading and cavitating upper-hybrid turbulence in the same way that the cascade and broad ISR spectra are the signatures of cascading and cavitating Langmuir turbulence (Mjølhus, 1998). Upper-hybrid turbulence is almost certainly, perhaps in conjunction with Langmuir turbulence, involved in the production of the energetic electrons which produce RF-induced airglow (Gustavsson et al., 2002; Kosch et al., 2002a, 2004; Istomin and Leyser, 2003).

Past angle-scanning observations have concentrated on the magnetic meridian, mainly between vertical and magnetic field-aligned. Very few observations have explored beyond field-aligned to the south, even fewer have explored north of vertical. Optical imaging observations may cover a large field-of-view in two dimensions, and observations of an airglow ring extending both along and across the meridian suggest that not only elevation scans but also azimuth 
scans might be of interest for ISR and other narrow-beam measurements.

Even wide-angle observations must consider scanning, by the pump beam, in order to illuminate regions of interest within the field-of-view. These include the interferometric SEE measurements discussed here along with wide-angle optical measurements. Circular scanning of the pump beam performed at HAARP by Pedersen et al. (2003) show optical emission enhancements only in the vicinity of the fieldaligned position. However, these observations were at relatively low ERP, similar to that used by Kosch et al. (2000). At roughly five times greater ERP, Kosch et al. (2002b, 2004) found not only that the airglow region formed a ring but that it followed the pump beam. The higher ERP is due mainly to the use of the EISCAT high-gain HF antenna, which has a relatively narrow 7.5-degree 3-dB full beamwidth. The higher power density and narrower beam imply higher RF electric field and power gradients within the beam, another factor which could be important in the formation and movement of the airglow ring. The frequency was also very close to the fourth gyroharmonic, which could be important as this was also different than past observations. Due to limitations in the transmitter hardware, cross-meridian pump scanning cannot be performed at EISCAT. However, when construction of the final HAARP array is complete, these experiments will be able to be repeated with scanning both along and across the meridian at ERPs of over $1 \mathrm{GW}$. Another location of interest for future observations is on Svalbard, where the SPEAR HF facility, located next to the EISCAT Svalbard Radar (ESR), was installed in 2003 and has been undergoing testing along with preliminary experimentation since April 2004 (Wright et al., 2000).

Differing conclusions have been reached regarding the role of upper-hybrid vs. Langmuir turbulence in artificial airglow production under conditions of significantly different ERP: upper-hybrid turbulence appeared to dominate with an ERP of about $110 \mathrm{MW}$ (Kosch et al., 2002a), and Langmuir turbulence appeared to be important when using an ERP of $550 \mathrm{MW}$ (Kosch et al., 2004). However, these observations were at the third and at or near the fourth gyroharmonic, and used low and high gain antennas, producing lesser and greater RF field and power gradients, respectively, differences which again could be important. Although thermal electrons may play a role in producing RF-pumpenhanced airglow, non-thermal electrons, and therefore most likely some form of turbulence, are still required to explain the observations, even during experiments at relatively low ERP (Gustavsson et al., 2002).

Even if Langmuir turbulence turns out to be the correct explanation for the airglow observations near the fourth gyroharmonic at high ERP, a mystery remains at low ERP. At the gyroharmonics many pump-induced effects, including electron temperature enhancements, anomalous absorption, artificial ionospheric irregularities, and upper-hybrid turbulence, are known to be minimized (Stubbe et al., 1994; Robinson et al., 1996; Honary et al., 1999; Ponomarenko et al.,
1999). Thus, the pump power which otherwise would be used to produce these effects should be available to produce Langmuir turbulence, and if Langmuir turbulence is a source of accelerated electrons, then airglow should be observed. However, artificial airglow is another effect that is known to be minimized at the gyroharmonics during low ERP experiments (Kosch et al., 2002a). This apparent paradox might be explained if for some reason Langmuir turbulence turns out to have a relatively high threshold for electron acceleration.

The preliminary data presented in Figs. 12, 13, and 14 hold great promise due in part to the nearness of the pump frequency to the fourth gyroharmonic, which allows a comparison between the $\mathrm{BUM}_{1}$ and DM features of the SEE spectrum. The BUM ${ }_{1}$ has been linked to Langmuir turbulence (Frolov and Nedzvedski, 2004; Frolov et al., 2004 ${ }^{1}$ ), and the pump-beam-following motion is consistent with the high-pump-power fourth-gyroharmonic (approximately $5.423 \mathrm{MHz}$ ) optical observations, also linked to Langmuir turbulence. By contrast the DM is linked to upper hybrid turbulence (Mjølhus, 1998; Leyser, 2001). The angles calculated from the present data show that both the BUM $\mathrm{B}_{1}$ and the DM may be equally affected by the HF beam direction change, although the DM appears to reside at higher elevation angle than the $\mathrm{BUM}_{1}$. These results are tentative. However, a difference in the aspect angle behavior of these features could have important implications for the underlying physics of the turbulence and for the explanation of past observations. The possible angle dependence of the $\mathrm{BUM}_{2}$, as suggested by the 12 November 2001 beam swinging data shown in Fig. 10, is also of interest in this regard and can be further investigated using the new data set. Final conclusions must await additional and complete processing and interpretation of the SEE and other available data.

The apparently repeatable, gradual decrease in the coherence of both the BUM ${ }_{1}$ and DM features could be due to an increase in the size of the source region during the pump-on period. This might be understood, for example, if the initial size were of the order of one or several Fresnel zones at the pump wavelength, increasing thereafter to encompass additional Fresnel zones, thus increasing destructive interference between neighboring locations within the source region and reducing the coherence. At $5.423 \mathrm{MHz}$, a Fresnel zone is of the order of $\left(\lambda_{\mathrm{hf}} r_{\mathrm{see}}\right)^{1 / 2}=[(55.32 \mathrm{~m})(180 \mathrm{~km})]^{1 / 2}=3.2 \mathrm{~km}$, where $\lambda_{\mathrm{hf}}$ is the free-space wavelength and $r_{\text {see }}$ is the range to the SEE source region as determined from EISCAT 224$\mathrm{MHz}$ radar measurements. An interesting and unexplained observation is the apparent dependence of the DM coherence on the orientation of the antenna baseline. The significance of this, and of the structure observed in the DM coherence plots, is currently unknown.

Stubbe and Hagfors (1997) comment: "One of the shortcomings in the theoretical work appears to be an inappropriate treatment of the low frequency turbulence which depends very sensitively on the direction with respect to the magnetic field, implying that the full angular spectrum rather than a prescribed fixed angular component should be considered.' Although Mjølhus et al. (2003) have recently addressed this 
criticism for the theoretical interpretation of incoherent scatter observations of Langmuir turbulence, much remains to be done. Furthermore, this comment applies equally well to experimental results. Pump-enhanced electron temperature, irregularities, airglow, and upper-hybrid and Langmuir turbulence are now experimentally known to be aspect-angle dependent. This further supports the contention that SEE must also be, and provides additional impetus for performing the angle-resolved SEE measurements discussed here.

\section{Conclusions}

The intensification of HF-enhanced Langmuir waves and airglow near the geomagnetic field-aligned direction suggest that stimulated electromagnetic emission might exhibit a similar angular dependence, possibly different for the different spectral features. In order to see if this suspicion holds true, a pilot experiment has been completed and data analysis is underway.

By combining two antenna pairs as interferometers with approximately north-south and east-west baselines, with antenna spacing on the order of a wavelength in the frequency band of interest, the cross-phase from each pair is being used to calculate the direction of the signal-weighted average of the SEE source region within the HF pump beam pattern. These results are valid in regions of high cross-spectral coherence. Preliminary calculations already suggest intriguing connections with past angle scanning results using other techniques.

In future observations, the use of two orthogonallypolarized antennas along each baseline would allow the study of possible polarization dependence of the results. Multiple antennas at multi-wavelength spacings might at some time be used to produce images of the SEE source region, thereby removing the signal-weighted limitation of the current technique.

It is our hope that, in this way, a wealth of new information will be obtained on the wave interaction and turbulent processes occurring in the pump-plasma interaction region. Such results promise to be a new and important resource in helping to identify SEE excitation mechanisms and their relation to the observational and theoretical properties of Langmuir and upper-hybrid turbulence.

Acknowledgements. The EISCAT Scientific Association is supported by Suomen Akatemia of Finland, Centre National de la Recherche Scientifique of France, Max-Planck-Gesellschaft of Germany, the National Institute of Polar Research of Japan, Norges Forskningsråd of Norway, Vetenskapsrådet of Sweden, and the Particle Physics and Astronomy Research Council of the United Kingdom. B. I. is grateful to Interamerican University of Puerto Rico, the EISCAT Scientific Association, the High Frequency Auroral Active Research Program, the Puerto Rico Alliance for Minority Participation, and the SNAP Development Center for supporting portions of this work. Support for B. K., R. Yu. Y., and E. D. T., and for development of the Polar Geophysical Institute receiver, was provided by INTAS grant 03-51-5583 and Russian Foundation of Fundamental Research grant 03-05-64636. We thank M. Lehtinen for the loan of an Invers Oy digital receiver, E. Mjølhus for interesting and insightful discussions, $\mathrm{H}$. Kohl for providing the original copy of the ray tracing program, and C. Otero for his assistance in producing Fig. 10. T. H. and C. L. H. proposed the experiment; B. I., C. H., and M. T. R. carried out initial trials using a digital receiver from Invers Oy; B. K., R. Yu. Y., and E. D. T. developed the Polar Geophysical Institute receiver in collaboration with T. H., B. I., M. T. R., and C. L. H.; B. I. and M. T. R. analyzed the data for Fig. 10; B. K. and R. Yu. Y. carried out the Sura observations and analyzed the data; A. B. supported, and B. K., R. Yu. Y., M. T. R., B. I., V. B., and M. G. carried out, the Ramfjordmoen observations; M. G. produced Ramfjordmoen SEE overview plots; V. B., B. I., B. K., R. Yu. Y., E. D. T., and T. H. analyzed the Ramfjordmoen SEE interferometer data; B. I. and T. H. wrote the paper and produced the final figures.

Topical Editor M. Lester thanks two referees for their help in evaluating this paper.

\section{References}

Belikovich, V. V., Benediktov, E. A., Tolmacheva, A. V., and Bakhmet'eva, N. V.: Ionospheric research by means of artificial periodic irregularities, English edition (translated by Förster, M. and Rietveld, M. T.), Copernicus GmbH (D-37191 KatlenburgLindau, Germany), ISBN 3-936586-03-9, 160 pages, 2002.

Bernhardt, P. A., Tepley, C. A., and Duncan, L. M.: Airglow enhancements associated with plasma cavities formed during ionospheric heating experiments, J. Geophys. Res., 94, 9071-9092, 1989.

Carlson, H. C., Wickwar, V. B., and Mantis, G. P.: Observations of fluxes of suprathermal electrons accelerated by HF-excited instabilities, J. Atmos. Terr. Phys., 44, 1089-1100, 1982.

Carozzi, T. D., Thidé, B., Leyser, T. B., Komrakov, G., Frolov, V., Grach, S., and Sergeev, E.: Full polarimetry measurements of stimulated electromagnetic emissions: First results, J. Geophys. Res., 106(A10), 21 395-21 407, 2001.

Carozzi, T. D., Thidé, B., Grach, S. M., Leyser, T. B., Holz, M., Komrakov, G. P., Frolov, V. L., and Sergeev, E. N.: Stimulated electromagnetic emissions during pump frequency sweep through fourth electron cyclotron harmonic, J. Geophys. Res., 107(A9), 1253, doi:10.1029/2001JA005082, 2002.

Dhillon, R. S. and Robinson, T. R.: Observations of time dependence and aspect sensitivity of regions of enhanced UHF backscatter associated with RF heating, Ann. Geophys., 23, 75$85,2005$.

Djuth, F. T., Bernhardt, P. A., Tepley, C. A., Gardner, J. A., Kelley, M. C., Broadfoot, A. L., Kagan, L. M., Sulzer, M. P., Elder, J. H., Selcher, C., Isham, B., Brown, C., and Carlson, H. C.: Large airglow enhancement produced via wave-plasma interactions in sporadic E, Geophys. Res. Lett., 26(11), 1557-1560, 1999.

Eliasson, B.: Numerical simulation of kinetic effects in ionospheric plasma, IT Licentiate Thesis 2001-004, Uppsala Univ. Dept. of Information Technology, available at http://www.physics.irfu.se/ Publications/, 2001.

Ellis, G. R.: F -region triple splitting, J. Atmos. Terr. Phys., 3, 263269, 1953.

Ellis, G. R.: The Z propagation hole in the ionosphere, J. Atmos. Terr. Phys., 8, 43-54, 1956.

Frolov, V. L., Erukhimov, L. M., Kagan, L. M, Komrakov, G. P., Sergeev, E. N., and Stubbe, P.: Two-component nature of the broad upshifted maximum in stimulated electromagnetic emission spectra, Phys. Rev. Lett., 81(8), 1630-1633, 1998. 
Frolov, V. L., Sergeev, E. N., Ermakova, E. N., and Komrakov, G. P.: Spectral features of stimulated electromagnetic emission measured in the 4.3-9.5 MHz pump wave frequency range, Geophys. Res. Lett., 28, 3103-3106, 2001.

Frolov, V. L.: Control of spectral characteristics of artificial lowfrequency ionosphere turbulence, Geomagn. Aeron., 42, 159$165,2003$.

Frolov, V. L. and Nedzvetski, D. I.: The study of the aspect angle dependence for some SEE components. VI International Suzdal Symposium ISS-04, Moscow, 19-21 October 2004, Book of Abstracts, 36, 2004.

Gurevich, A. V., Carlson, H. C., Milikh, G. M., Zybin, K. P., Djuth, F. T., and Groves, K.: Suprathermal electrons generated by the interaction of powerful radio wave with the ionosphere, Geophys. Res. Lett., 27(16), 2461-2464, 2000.

Gurevich, A. V., Zybin, K. P., Carlson, H. C., and Pedersen, T.: Magnetic zenith effect in ionospheric modifications, Phys. Lett. A, 305, 264-274, 2002.

Gustavsson, B., Brändström, B. U. E., Steen, Å., Sergienko, T., Leyser, T. B., Rietveld, M. T., Aso, T., and Ejiri, M.: Nearly simultaneous images of HF-pump enhanced airglow at $6300 \AA$ and $5577 \AA$, Geophys. Res. Lett., 29(24), 2220, doi:10.1029/2002GL015350, 2002.

Honary, F., Robinson, T. R., Wright, D. M., Stocker, A. J., Rietveld, M. T., and McCrea, I.: First direct observations of the reduced striations at pump frequencies close to the electron gyroharmonics, Ann. Geophys., 17, 1235-1238, 1999,

SRef-ID: 1432-0576/ag/1999-17-1235.

Isham, B., Kofman, W., Hagfors, T., Nordling, J., Thidé, B., La Hoz, C., and Stubbe, P.: New phenomena observed by EISCAT during an RF ionospheric modification experiment, Radio Sci., 25, 251-262, 1990.

Isham, B., Hagfors, T., Mishin, E., Rietveld, M. T., La Hoz, C., Kofman, W. and Leyser, T.: A search for the location of the HF excitation of enhanced ion acoustic and Langmuir waves with EISCAT and the Troms $\varnothing$ heater, Radiophys. Quant. Elec. (Russian orig.), 42(7), 607-618, 1999a.

Isham, B., La Hoz, C., Rietveld, M. T., Hagfors, T., and Leyser, T. B.: Cavitating Langmuir turbulence observed during high latitude ionospheric wave interaction experiments, Phys. Res. Lett., 83, 13, 2576-2579, 1999b.

Isham, B., Rietveld, M. T., Hagfors, T., La Hoz, C., Mishin, E., Kofman, W., Leyser, T. B., and van Eyken, A. P.: Aspect angle dependence of HF enhanced incoherent backscatter, Adv. Space Res., 24, 1003-1006, 1999c.

Isham, B., Tepley, C. A., Sulzer, M. P., Zhou, Q. H., Kelley, M. C., Friedman, J. S., and González, S. A.: Upper atmospheric observations at the Arecibo Observatory: Examples obtained using new capabilities, J. Geophys. Res., 105(A8), 18 609-18637, 2000.

Istomin, Ya. N. and Leyser, T. B.: Electron acceleration by cylindrical upper-hybrid oscillations trapped in density irregularities in the ionosphere, Phys. Plasmas, 10(7), 2962-2970, 2003.

Jussila, J.: Aurora - Revontulien Taivaallinen Näytelmä (English title: Aurora - Heavenly Display of Northern Lights, text in Finnish with English summary), Sanoma WSOY (Helsinki, Finland), ISBN 951-0-27451-8, 128, 2002.

Kosch, M. J., Rietveld, M. T., Hagfors, T., and Leyser, T. B.: Highlatitude HF-induced airglow displaced equatorward of the pump beam, Geophys. Res. Lett., 27, 2817-2820, 2000.

Kosch, M. J., Rietveld, M. T., Kavanagh, A. J., Davis, C., Yeoman, T. K., Honary, F., and Hagfors, T.: High-latitude pump- induced optical emissions for frequencies close to the third electron gyroharmonic, Geophys. Res. Lett., 29(23), 2112, doi:10.1029/2002GL015744, 2002a.

Kosch, M. J., Rietveld, M. T., Kavanagh, A. J., Jussila, J., Isham, B., Kaila, K., McCrea, I. W., Senior, A., Holma, H., Honary, F., Hagfors, T.: High-latitude artificial aurora using the EISCAT highgain HF facility, Proc. URSI 27th General Assembly, 2002b.

Kosch, M. J., Rietveld, M. T., Senior, A., McCrea, I. W., Kavanagh, A. J., Isham, B., and Honary, F.: Novel artificial optical annular structures in the high latitude ionosphere over EISCAT, Geophys. Res. Lett., 31, L12805, 1-4, doi:10.1029/2004GL019713, 2004.

Leyser, T. B., Thidé, B., Derblom, H., Hedberg, Å., Lundborg, B., Stubbe, P., and Kopka, H.: Stimulated electromagnetic emission near electron cyclotron harmonics in the ionosphere, Phys. Rev. Lett., 63(11), 1145-1147, 1989.

Leyser, T. B.: Stimulated electromagnetic emissions by highfrequency electromagnetic pumping of the ionospheric plasma, Space Sci. Rev., 98, 223-328, 2001.

Lundborg, B. and Thidé, B.: Standing wave pattern of HF radio waves in the ionospheric reflection region, 1, general formulas, Radio Sci., 20(4), 947-958, 1985.

Lundborg, B. and Thidé, B.: Standing wave pattern of HF radio waves in the ionospheric reflection region, 2, applications, Radio Sci., 21(3), 486-500, 1986.

Mishin, E., Hagfors, T., and Isham, B.: A generation mechanism for topside enhanced incoherent backscatter during high frequency modification experiments in Troms $\varnothing$, Geophys. Res. Lett., 28(3), 479-482, 2001.

Mjølhus, E.: On linear conversion in a magnetized plasma, Radio Sci., 25(6), 1321-1339, 1990.

Mjølhus, E.: Theoretical model for long time stimulated electromagnetic emission generation in ionospheric radio modification experiments, J. Geophys. Res., 103(A7), 3, 14711-14729, 1998.

Mjølhus, E. and Flå, T.: Direct access to plasma resonance in ionospheric radio experiments, J. Geophys. Res., 89(A6), 3921-3928, 1984.

Mjølhus, E., Helmersen, E., and DuBois, D. F.: Geometric aspects of HF-driven Langmuir turbulence in the ionosphere, Nonlin. Proc. Geophys., 10, 151-177, 2003,

\section{SRef-ID: 1607-7946/npg/2003-10-151.}

Pedersen, T. R., McCarrick, M., Gerken, E., Selcher, C., Sentman, D., Carlson, H. C., and Gurevich, A.: Magnetic zenith enhancement of HF radio-induced airglow production at HAARP, Geophys. Res. Lett., 30(4), 1169, doi:10.1029/2002GL016096, 2003.

Ponomarenko, P. V., Leyser, T. B., and Thidé, B.: New electron gyroharmonic effects in HF scatter from pump excited magnetic field-aligned ionospheric irregularities, J. Geophys. Res., 104(A5), 10081-10087, 1999.

Revenchuk, S., Thidé, B., and Pavlenko, V.: Wave tunneling in the ionosphere due to plasma kinetic effects, 1, Collisionless limit, and 2, Influence of collisions, preprints available at http://www. physics.irfu.se/Publications/, 2001.

Rietveld, M. T., Kohl, H., Kopka, H., and Stubbe, P.: Introduction to ionospheric heating at Troms $\varnothing, 1$, Experimental overview, J. Atmos. Terr. Phys., 55, 2, 577-599, 1993.

Rietveld, M. T., Kosch, M. J., Blagoveshchenskaya, N. F., Kornienko, V. A., Leyser, T. B., and Yeoman, T. K.: Ionospheric electron heating, optical emissions, and striations induced by powerful HF radio waves at high latitudes: Aspect angle dependence, J. Geophys. Res., 108(A4), 1141 , doi:10.1029/2002JA009543, 2003. 
Robinson, T. R., Honary, F., Stocker, A. J., Jones, T. B., and Stubbe, P.: First EISCAT observations of the modification of F-region electron temperatures during RF heating at harmonics of the electron gyrofrequency, J. Atmos. Terr. Phys., 58(1-4), 385-395, 1996.

Robinson, P. A.: Nonlinear wave collapse and strong turbulence, Rev. Mod. Phys., 69, 2, 507-573, 1997.

Seliga, T. A.: Ionospheric heating via ordinary to extraordinary mode wave coupling, Radio Sci., 20(3), 565-574, 1985.

Stolarski, R. S., Dulock Jr., V. A., Watson, C. E., and Green, A. E. S.: Electron impact cross sections for atmospheric species, 2, molecular nitrogen, J. Geophys. Res., 72(15), 3953-3960, 1967.

Stubbe, P. and Hagfors, T.: The earth's ionosphere: A wall-less plasma laboratory, Surv. Geophys., 18(1), 57-127, 1997.

Stubbe, P. and Kopka, H.: Stimulated electromagnetic emission in a magnetized plasma: A new symmetric spectral feature, Phys. Rev. Lett., 65, 183-186, 1990.
Stubbe, P., Kopka, H., Thidé, B., and Derblom, H.: Stimulated electromagnetic emission: a new technique to study the parametric instability in the ionosphere, J. Geophys. Res., 89(A9), 75237536, 1984.

Stubbe, P., Stocker, A. J., Honary, F., Robinson, T. R., and Jones, T. B.: Stimulated electromagnetic emissions and anomalous HF wave absorption near electron gyroharmonics, J. Geophys. Res., 99(A4), 6233-6246, 1994.

Thidé, B., Kopka, H., and Stubbe, P.: Observations of stimulated scattering of a strong high-frequency radio wave in the ionosphere, Phys. Rev. Lett., 49, 1561-1564, 1982.

Wright, D. M., Davies, J. A., Robinson, T. R., Chapman, P. J., Yeoman, T. K., Thomas, E. C., Lester, M., Cowley, S. W. H., Stocker, A. J., Horne, R. B., and Honary, F.: Space Plasma Exploration by Active Radar (SPEAR): an overview of a future radar facility, Ann. Geophys., 18, 1248-1255, 2000,

SRef-ID: 1432-0576/ag/2000-18-1248. 